\title{
THE OUTBURST DECAY OF THE LOW MAGNETIC FIELD MAGNETAR SGR 0418+5729
}

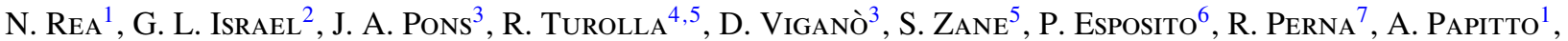

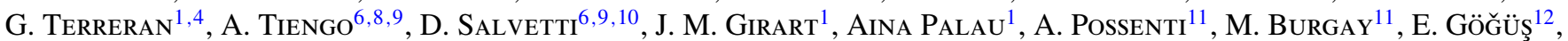 \\ G. A. Caliandro ${ }^{1}$, C. Kouveliotou ${ }^{13}$, D. Götz ${ }^{14}$, R. P. Mignan ${ }^{5,6,15}$, E. Ratti ${ }^{16}$, and L. Stella ${ }^{2}$ \\ ${ }^{1}$ Institute of Space Sciences (CSIC-IEEC), Campus UAB, Faculty of Science, Torre C5-parell, E-08193 Barcelona, Spain \\ ${ }^{2}$ INAF/Osservatorio Astronomico di Roma, via Frascati 33, I-00040 Monteporzio Catone, Italy \\ ${ }^{3}$ Department de Fisica Aplicada, Universitat d'Alacant, Ap. Correus 99, E-03080 Alacant, Spain \\ ${ }^{4}$ Dipartimento di Fisica e Astronomia, Università di Padova, via F. Marzolo 8, I-35131 Padova, Italy \\ ${ }^{5}$ Mullard Space Science Laboratory, University College London, Holmbury St. Mary, Dorking, Surrey RH5 6NT, UK \\ ${ }^{6}$ INAF/Istituto di Astrofisica Spaziale e Fisica Cosmica-Milano, via E. Bassini 15, I-20133 Milano, Italy \\ 7 JILA and Department of Astrophysical and Planetary Sciences, University of Colorado, Boulder, CO 80309, USA \\ ${ }^{8}$ IUSS-Istituto Universitario di Studi Superiori, Piazza della Vittoria 15, I-27100 Pavia, Italy \\ ${ }^{9}$ INFN Istituto Nazionale di Fisica Nucleare, Sezione di Pavia, Via Bassi 6, I-27100 Pavia, Italy \\ ${ }^{10}$ Dipartimento di Fisica Nucleare e Teorica, Università degli Studi di Pavia, via Bassi 6, I-27100 Pavia, Italy \\ ${ }^{11}$ INAF/Osservatorio Astronomico di Cagliari, località Poggio dei Pini, strada 54, I-09012 Capoterra, Italy \\ 12 Sabancı University, Orhanl1-Tuzla, 34956 İstanbul, Turkey \\ ${ }^{13}$ NASA Marshall Space Flight Center, Huntsville, AL 35812, USA \\ 14 AIM (UMR 7158 CEA/DSM-CNRS-Université Paris Diderot) Irfu/Service d'Astrophysique, Saclay, F-91191 Gif-sur-Yvette Cedex, France \\ ${ }_{15}^{15}$ Kepler Institute of Astronomy, University of Zielona Góra, Lubuska 2, 65-265 Zielona Góra, Poland \\ ${ }^{16}$ SRON-Netherlands Institute for Space Research, Sorbonnelaan 2, 3584 CA Utrecht, The Netherlands \\ Received 2013 January 23; accepted 2013 April 16; published 2013 May 24
}

\begin{abstract}
We report on the long-term X-ray monitoring of the outburst decay of the low magnetic field magnetar SGR 0418+5729 using all the available X-ray data obtained with RXTE, Swift, Chandra, and XMM-Newton observations from the discovery of the source in 2009 June up to 2012 August. The timing analysis allowed us to obtain the first measurement of the period derivative of SGR 0418+5729: $\dot{P}=4(1) \times 10^{-15} \mathrm{~s} \mathrm{~s}^{-1}$, significant at $\mathrm{a} \sim 3.5 \sigma$ confidence level. This leads to a surface dipolar magnetic field of $B_{\text {dip }} \simeq 6 \times 10^{12} \mathrm{G}$. This measurement confirms SGR 0418+5729 as the lowest magnetic field magnetar. Following the flux and spectral evolution from the beginning of the outburst up to $~ 1200$ days, we observe a gradual cooling of the tiny hot spot responsible for the X-ray emission, from a temperature of $\sim 0.9$ to $0.3 \mathrm{keV}$. Simultaneously, the X-ray flux decreased by about three orders of magnitude: from about $1.4 \times 10^{-11}$ to $1.2 \times 10^{-14} \mathrm{erg} \mathrm{s}^{-1} \mathrm{~cm}^{-2}$. Deep radio, millimeter, optical, and gamma-ray observations did not detect the source counterpart, implying stringent limits on its multi-band emission, as well as constraints on the presence of a fossil disk. By modeling the magneto-thermal secular evolution of SGR 0418+5729, we infer a realistic age of $\sim 550 \mathrm{kyr}$, and a dipolar magnetic field at birth of $\sim 10^{14} \mathrm{G}$. The outburst characteristics suggest the presence of a thin twisted bundle with a small heated spot at its base. The bundle untwisted in the first few months following the outburst, while the hot spot decreases in temperature and size. We estimate the outburst rate of low magnetic field magnetars to be about one per year per galaxy, and we briefly discuss the consequences of such a result in several other astrophysical contexts.
\end{abstract}

Key words: stars: individual (SGR 0418+5729) - stars: magnetic field - stars: neutron

Online-only material: color figures

\section{INTRODUCTION}

Neutron stars showing magnetar-like activity (comprising the anomalous X-ray pulsars, soft gamma repeaters, and a high magnetic field pulsar) are a small group of X-ray pulsars (about 20 objects) with spin periods between $0.3-12 \mathrm{~s}$, whose strong persistent and/or flaring emission are hard to explain using the common scenarios for rotation-powered pulsars or accreting pulsars. In fact, the very strong X-ray emission of these objects $\left(L_{\mathrm{X}} \sim 10^{35} \mathrm{erg} \mathrm{s}^{-1}\right)$ is too high and/or variable to be fed by the rotational energy alone (as in the radio pulsars), and no evidence for a companion star has been found, hence ruling out accretion in a binary. Accretion from a fossil disk remnant of the supernova explosion might be responsible for part of the observational properties of these objects, but it fails to explain some of their characteristics, such as the flaring X-ray activity. Their inferred magnetic fields, under the typical assumption of magnetic dipolar losses alone, appear to be as high as $B_{\text {dip }} \simeq 3.2 \times 10^{19} \sqrt{P \dot{P}} \sim 10^{14}-10^{15} \mathrm{G}$ (see Mereghetti
2008 for a review). These strong fields are believed to either form via a dynamo action in a rapidly rotating protoneutron star $(<3 \mathrm{~ms}$; Thompson \& Duncan 1995) or be fossil field remnants of a highly magnetic massive star $(\sim 1 \mathrm{kG}$; Ferrario \& Wickramasinghe 2006). Because of these high $B$ fields, the emission of "magnetars" is thought to be powered by the decay and the instability of their strong fields (Duncan \& Thompson 1992; Thompson \& Duncan 1993; Thompson et al. 2002). Their powerful X-ray output is usually well modeled by thermal emission from the neutron star hot surface, reprocessed in a twisted magnetosphere through resonant cyclotron scattering (Thompson et al. 2002; Nobili et al. 2008; Rea et al. 2008; Zane et al. 2009), a process favored under these extreme magnetic conditions. On top of their persistent X-ray emission, magnetars emit very peculiar flares and outbursts on several timescales, from fractions of a second to years reaching very high, super-Eddington luminosities $\left(10^{38}-10^{46} \mathrm{erg} \mathrm{s}^{-1}\right)$. These flares are most probably caused by rearrangements of the twisted magnetic field lines, either accompanied or triggered by 
fractures of the neutron-star crust (Thompson \& Duncan 1995; Perna \& Pons 2011).

Transient events are a characteristic signature of magnetar emission, and are one of the main ways to discover new sources of this class and study its physics. From the discovery of the first transient less than a decade ago, we now count about a dozen outbursts, which increased the number of known magnetars by a third in six years (see Rea \& Esposito 2011; Rea 2013 for recent reviews). Magnetar outbursts might involve their multiband emission resulting in an increased activity from the radio to hard X-ray, usually with a soft X-ray flux increase of a factor of $10-1000$ with respect to the quiescent level. An associated X-ray spectral evolution is often observed, with a spectral softening during the outburst decay (Rea et al. 2009). The flux decay timescale varies substantially from source to source, ranging from a few weeks to several years (Rea \& Esposito 2011; Pons \& Rea 2012).

The extensive follow-up of magnetars undergoing an outburst yielded the most unexpected discovery of recent years in the magnetar field. Prompted by the detection of typical magnetarlike bursts and a powerful outburst, a new transient magnetar with a spin period of $\sim 9 \mathrm{~s}$ was discovered in 2009, namely SGR 0418+5729 (van der Horst et al. 2010; Esposito et al. 2010). However, after more than two years of extensive monitoring, no period derivative was detected. This led to an upper limit on the source surface dipolar field of $B_{\text {dip }}<7.5 \times 10^{12} \mathrm{G}$ (Rea et al. 2010). For the first time, we detected a magnetar with a low dipolar magnetic field, showing that a critical magnetic field is not necessary for a neutron star in order to display magnetar-like activity. In turn, this means that many seemingly normal pulsars could turn out to be magnetars at anytime (this was supported by the discovery of a second low- $B$ magnetar following soon after that of the first; Rea et al. 2012; Scholz et al. 2012). After the discovery of this low dipolar magnetic field soft gamma repeater, several models were put forward to explain its puzzling emission. They involve the possible presence of a fallback disk slowing down the pulsar up to the current spin period (Alpar et al. 2011), a tiny inclination angle between the magnetic and rotational axis resulting in a higher inferred magnetic field (Tong \& Xu 2012), a pulsar with a strongly magnetized core (Soni 2012), an old quark nova (Ouyed et al. 2011), or a massive highly magnetized, rotating white dwarf (Malheiro et al. 2012). In Rea et al. (2010) and Turolla et al. (2011), we suggested that a non-dipolar component of the field, larger than the measured dipolar one, can be responsible for the behavior of this magnetar, if it has a relatively old age ( $\simeq 1$ Myr).

In this paper we present the complete study of the outburst of the low dipolar magnetic field magnetar SGR 0418+5729, from the first outburst phases until about three years after its onset. This long-term monitoring campaign using several X-ray satellites, allowed us to estimate SGR 0418+5729's period derivative, and follow the cooling of its surface temperature during the outburst decay up to the (probable) quiescent level. Furthermore, we inferred limits on its emission in the radio, millimeter, optical, and gamma-ray bands. We discuss our findings in terms of the magneto-thermal history of this magnetar, discuss the current limits on the presence of a fossil disk, and present some discussion on the broader consequences of the discovery of low magnetic field magnetars.

\section{X-RAY OBSERVATIONS AND DATA REDUCTION}

In this study, we used data obtained from several different satellites (see Table 1 and Figure 1 for a summary). We describe below the observations and data analysis. Parts of the data we used in this paper were already published by van der Horst et al. (2010), Esposito et al. (2010), and Rea et al. (2010).

\subsection{Swift Data}

The X-Ray Telescope (XRT; Burrows et al. 2005) on board Swift uses a front-illuminated CCD detector sensitive to photons between 0.2 and $10 \mathrm{keV}$. Two main readout modes are available: photon counting (PC) and windowed timing (WT). PC mode provides two-dimensional imaging information and a $2.5073 \mathrm{~s}$ time resolution; in WT mode only one-dimensional imaging is preserved, achieving a time resolution of $1.766 \mathrm{~ms}$. The XRT data were uniformly processed with XRTPIPELINE (version 12, in the HEASOFT software package version 6.11), and filtered and screened with standard criteria, correcting for effective area, dead columns, etc. The source counts were extracted within a 20 pixel radius (one XRT pixel corresponds to about 2 '.36). For the spectroscopy, we used the spectral redistribution matrices in CALDB (20091130; matrices version v013 and v014 for the PC and WT data, respectively), while the ancillary response files were generated with XRTMKARF, and they account for different extraction regions, vignetting, and point-spread function (PSF) corrections.

\subsection{RXTE Data}

The Proportional Counter Array (PCA; Jahoda et al. 1996) on board RXTE consists of five collimated xenon/methane multi-anode Proportional Counter Units (PCUs) operating in the $2-60 \mathrm{keV}$ energy range. Raw data were reduced using the FTOOLs package (version 6.11). To study the timing properties of SGR 0418+5729, we restricted our analysis to the data in Good Xenon mode, with a time resolution of $1 \mu$ s and 256 energy bins. The event-mode data were extracted in the $2-10 \mathrm{keV}$ energy range from all active detectors (in a given observation) and all layers, and binned into light curves of $0.1 \mathrm{~s}$ resolution. We use here 46 RXTE/PCA observations of SGR 0418+5729, spanning the first six months of the outburst, until the source flux decayed below the instrument detection level. The total $194.2 \mathrm{ks}$ exposure time is divided in observations of 0.6 to $13.6 \mathrm{ks}$ exposure each. See Esposito et al. (2010) for further details on the Swift and $R X T E$ observations.

\subsection{Chandra Data}

The Chandra X-Ray Observatory monitored SGR 0418+5729 five times during the past three years. The first observation was with the High Resolution Imaging Camera (HRC-I; Zombeck et al. 1995) and the following four observations were with the Advanced CCD Imaging Spectrometer (ACIS-S; Garmire et al. 2003). Data were analyzed using standard cleaning procedures ${ }^{17}$ and CIAO version 4.4. The HRC-I camera does not have a sufficient spectral resolution, and it was used only for the timing analysis; it has a timing resolution of $\sim 16 \mu \mathrm{s}$. All ACIS-S observations were performed in VERY FAINT mode, with only the $\mathrm{S} 7 \mathrm{CCD}$ on, resulting in a timing resolution of $0.44 \mathrm{~s}$. Photons were extracted from a circular region with a radius of $3^{\prime \prime}$ around the source position, including more than $90 \%$ of the source photons, and background was extracted from a similar region far from the source position.

\footnotetext{
$\overline{17 \text { http://asc.harvard.edu/ciao/threads/index.html }}$
} 
Table 1

Journal of All the X-Ray Observations of SGR 0418+5729

\begin{tabular}{|c|c|c|c|c|c|c|c|}
\hline Instrument & ObsID & Starting Date & $\begin{array}{c}\text { Exp. } \\
(\mathrm{ks})\end{array}$ & Counts s $^{-1}$ & Flux $^{\mathrm{c}}$ & $\begin{array}{l}k T_{\mathrm{BB}}{ }^{\mathrm{d}} \\
(\mathrm{keV})\end{array}$ & BB Norm. ${ }^{\mathrm{e}}$ \\
\hline$R X T E / \mathrm{PCA}^{\mathrm{a}}$ & 94048 & 2009 06-11/11-24 & 194.2 & & $\cdots$ & $\ldots$ & $\cdots$ \\
\hline Swift/XRT & 00031422001 & 2009-07-08 20:48:01 & 2.9 & $0.229 \pm 0.008$ & $13.4 \pm 1.0$ & $0.88 \pm 0.05$ & $2.2 \pm 0.2$ \\
\hline Swift/XRT (PC) & 00031422002 & 2009-07-09 00:04:01 & 10.6 & $0.245 \pm 0.003$ & $13.8 \pm 0.7$ & $0.94 \pm 0.03$ & $1.7 \pm 0.1$ \\
\hline Swift/XRT (PC) & 00031422003 & 2009-07-10 00:15:01 & 5.6 & $0.179 \pm 0.005$ & $11.0 \pm 0.7$ & $0.95 \pm 0.05$ & $1.33 \pm 0.13$ \\
\hline Swift/XRT (WT) & 00031422004 & 2009-07-12 00:27:01 & 7.1 & $0.218 \pm 0.006$ & $11.5 \pm 0.7$ & $0.91 \pm 0.04$ & $1.68 \pm 0.14$ \\
\hline Chandra/HRC-I ${ }^{\mathrm{a}}$ & 10168 & 2009-07-12 06:06:43 & 24.1 & $0.317 \pm 0.005$ & $\ldots$ & $\ldots$ & $\ldots$ \\
\hline Swift/XRT (WT) & 00031422006 & 2009-07-15 00:48:39 & 7.7 & $0.252 \pm 0.006$ & $12.7 \pm 1.0$ & $0.93 \pm 0.03$ & $1.69 \pm 0.12$ \\
\hline Swift/XRT (WT) & 00031422007 & 2009-07-16 00:53:01 & 16.4 & $0.217 \pm 0.004$ & $11.7 \pm 0.8$ & $0.93 \pm 0.02$ & $1.57 \pm 0.08$ \\
\hline XMM-Newton/EPIC* & 0610000601 & 2009-08-12 21:09:12 & 67.1 & $1.281 \pm 0.005$ & $6.75 \pm 0.07$ & $0.897 \pm 0.007$ & $1.038 \pm 0.018$ \\
\hline Swift/XRT (PC) & 00031422008 & 2009-09-20 21:09:00 & 9.4 & $0.066 \pm 0.002$ & $3.66 \pm 0.30$ & $0.82 \pm 0.05$ & $0.79 \pm 0.09$ \\
\hline Swift/XRT (PC) & 00031422009 & 2009-09-22 00:43:00 & 7.6 & $0.072 \pm 0.003$ & $3.58 \pm 0.40$ & $0.82 \pm 0.05$ & $0.79 \pm 0.11$ \\
\hline Swift/XRT (PC) & 00031422010 & 2009-11-08 00:36:01 & 15.1 & $0.043 \pm 0.002$ & $2.14 \pm 0.20$ & $0.82 \pm 0.05$ & $0.47 \pm 0.05$ \\
\hline Swift/XRT (PC) & $00031422011^{\mathrm{b} 1}$ & 2010-01-14 08:06:01 & 3.6 & $0.019 \pm 0.001^{\mathrm{b}}$ & $1.05 \pm 0.10$ & $0.75 \pm 0.07$ & $0.32 \pm 0.06$ \\
\hline Swift/XRT (PC) & $00031422012^{\mathrm{b} 1}$ & 2010-01-15 13:08:01 & 3.7 & $0.019 \pm 0.001^{\mathrm{b}}$ & $"$ & $"$ & $"$ \\
\hline Swift/XRT (PC) & $00031422013^{\mathrm{b} 1}$ & 2010-01-16 08:14:01 & 4.0 & $0.019 \pm 0.001^{\mathrm{b}}$ & $"$ & " & $"$ \\
\hline Swift/XRT (PC) & $00031422014^{\mathrm{b} 1}$ & 2010-01-17 06:47:01 & 3.8 & $0.019 \pm 0.001^{\mathrm{b}}$ & $"$ & " & $"$ \\
\hline Swift/XRT (PC) & $00031422015^{\mathrm{b} 2}$ & 2010-02-14 17:33:01 & 4.5 & $0.0172 \pm 0.0008^{\mathrm{c}}$ & $0.76 \pm 0.11$ & $0.74 \pm 0.04$ & $0.25 \pm 0.03$ \\
\hline Swift/XRT (PC) & $00031422016^{\mathrm{b} 2}$ & 2010-02-15 17:37:01 & 4.5 & $0.0172 \pm 0.0008^{c}$ & $"$ & " & $"$ \\
\hline Swift/XRT (PC) & $00031422017^{\mathrm{b} 2}$ & 2010-02-16 01:38:01 & 4.6 & $0.0172 \pm 0.0008^{\mathrm{c}}$ & $"$ & $"$ & $"$ \\
\hline Swift/XRT (PC) & $00031422018^{\mathrm{b} 2}$ & 2010-02-17 09:49:01 & 4.6 & $0.0172 \pm 0.0008^{c}$ & $"$ & ", & $"$ \\
\hline Swift/XRT (PC) & $00031422019^{\mathrm{b} 2}$ & 2010-02-18 16:14:01 & 3.9 & $0.0172 \pm 0.0008^{c}$ & $"$ & $"$ & $"$ \\
\hline Swift/XRT (PC) & $00031422020^{\mathrm{b} 2}$ & 2010-02-19 00:23:01 & 3.2 & $0.0172 \pm 0.0008^{\mathrm{c}}$ & $"$ & $"$ & $"$ \\
\hline Swift/XRT (PC) & $00031422021^{\mathrm{b} 3}$ & 2010-07-09 06:50:01 & 3.6 & $0.0023 \pm 0.0003^{\mathrm{d}}$ & $0.10 \pm 0.04$ & $0.47 \pm 0.13$ & $0.23 \pm 0.13$ \\
\hline Swift/XRT (PC) & $00031422022^{\mathrm{b} 3}$ & 2010-07-10 18:11:00 & 5.2 & $0.0023 \pm 0.0003^{\mathrm{d}}$ & $\bar{\prime}$ & $\overline{ }$ & $\bar{l}$ \\
\hline Swift/XRT (PC) & $00031422023^{\mathrm{b} 3}$ & 2010-07-11 05:19:01 & 5.0 & $0.0023 \pm 0.0003^{\mathrm{d}}$ & $"$ & $"$ & $"$ \\
\hline Swift/XRT (PC) & $00031422024^{\mathrm{b} 3}$ & 2010-07-11 23:06:01 & 5.4 & $0.0023 \pm 0.0003^{\mathrm{d}}$ & $"$ & " & $"$ \\
\hline Swift/XRT (PC) & $00031422025^{\mathrm{b} 3}$ & 2010-07-13 00:47:01 & 4.9 & $0.0023 \pm 0.0003^{\mathrm{d}}$ & $"$ & " & $"$ \\
\hline Chandra/ACIS-S & 12312 & 2010-07-23 15:04:09 & 30.0 & $0.0017 \pm 0.0008$ & $0.13 \pm 0.02$ & $0.68 \pm 0.04$ & $0.061 \pm 0.008$ \\
\hline XMM-Newton/EPIC & 0605852201 & 2010-09-24 01:54:56 & 34.2 & $0.0370 \pm 0.0020$ & $0.16 \pm 0.02$ & $0.69 \pm 0.05$ & $0.07 \pm 0.01$ \\
\hline Chandra/ACIS-S & 13148 & 2010-11-29 05:59:57 & 30.0 & $0.0038 \pm 0.0004$ & $0.021 \pm 0.002$ & $0.38 \pm 0.11$ & $0.12 \pm 0.06$ \\
\hline XMM-Newton/EPIC & 0672670201 & 2011-03-10 03:15:53 & 35.0 & $0.0071 \pm 0.0007$ & $0.015 \pm 0.002$ & $0.32 \pm 0.05$ & $0.21 \pm 0.08$ \\
\hline Chandra/ACIS-S & 13235 & 2011-07-20 02:26:12 & 77.0 & $0.0033 \pm 0.0002$ & $0.015 \pm 0.003$ & $0.37 \pm 0.04$ & $0.11 \pm 0.02$ \\
\hline XMM-Newton/EPIC ${ }^{\mathrm{b} 4}$ & 0672670401 & 2011-09-09 15:27:23 & 33.0 & $0.0071 \pm 0.0006^{\mathrm{e}}$ & $0.016 \pm 0.002$ & $0.28 \pm 0.05$ & $0.34 \pm 0.13$ \\
\hline XMM-Newton/EPIC ${ }^{\mathrm{b} 4}$ & 0672670501 & 2011-09-11 21:47:41 & 48.5 & $0.0071 \pm 0.0006^{\mathrm{e}}$ & $"$ & " & " \\
\hline Chandra/ACIS-S & 13236 & 2011-11-26 11:48:02 & 75.0 & $0.0026 \pm 0.0002$ & $0.015 \pm 0.002$ & $0.35 \pm 0.07$ & $0.13 \pm 0.05$ \\
\hline XMM-Newton/EPIC* & 0693100101 & 2012-08-25 14:18:08 & 78.2 & $0.0058 \pm 0.0004$ & $0.012 \pm 0.001$ & $0.32 \pm 0.05$ & $0.16 \pm 0.05$ \\
\hline
\end{tabular}

Notes.

a The RXTE-PCA and the Chandra HRC-I were used only for the timing analysis.

$\mathrm{b}$ These observations were merged in the timing and spectral analysis to improve statistics.

${ }^{c}$ Absorbed flux in the $0.5-10 \mathrm{keV}$ energy range, and in units of $10^{-12} \mathrm{erg} \mathrm{s}^{-1} \mathrm{~cm}^{-2}$. Errors in the table are at $90 \%$ confidence level.

${ }^{\mathrm{d}}$ Fitted model is phabs*bbodyrad; $N_{\mathrm{H}}=(1.15 \pm 0.06) \times 10^{21} \mathrm{~cm}^{-2}$ and $\chi_{v}^{2}=1.19$ (for $940 \mathrm{dof}$ ).

$\mathrm{e}$ The $\mathrm{BB}$ radius in $\mathrm{km}$ is the square root of this $\mathrm{BB}$ normalization, times the distance in units of $10 \mathrm{kpc}$.

* See Section 3.1 for details on the modeling of these observations.

\subsection{XMM-Newton Data}

SGR 0418+5729 was observed six times with XMM-Newton (Jansen et al. 2001). Data have been processed using SAS version 12 , and we have employed the most up-to-date calibration files available at the time the reduction was performed (2012 August). Standard data screening criteria are applied in the extraction of scientific products. For our spectral analysis we used only the EPIC-pn camera (Turner et al. 2001) which provides the spectra with the best statistics, while the MOS cameras (Strüder et al. 2001) were added in the timing analysis. The EPIC-pn camera was set in Small Window (timing resolution of $6 \mathrm{~ms}$ ) and Full Frame (73 ms) modes in the first two observations, respectively, and in Large Window mode for all the following ones $(48 \mathrm{~ms})$, with the source at the aim-point of the camera, and the MOS cameras in Small Window mode $(0.3 \mathrm{~s})$. We extracted the source photons from a circular region of $30^{\prime \prime}$ radius, and a similar region was chosen for the background in the same CCD. We restricted our spectral analysis to photons having PATTERN $\leqslant 4$ and FLAG $=0$ for the EPIC-pn data.

\section{RESULTS OF THE X-RAY MONITORING}

\subsection{X-Ray Spectral Modeling}

For the spectral analysis we used source and background photons from the Swift, Chandra, and XMM-Newton observations extracted as described in the previous section (we also checked our results using a larger extraction region for the background spectra). The response matrices were built using ad hoc bad-pixel files built for each observation. We used the XSPEC package (version 12.4) for all fittings, and the phabs absorption model with the Anders \& Grevesse (1989) abundances, and the Balucinska-Church \& McCammon (1992) photoelectric 

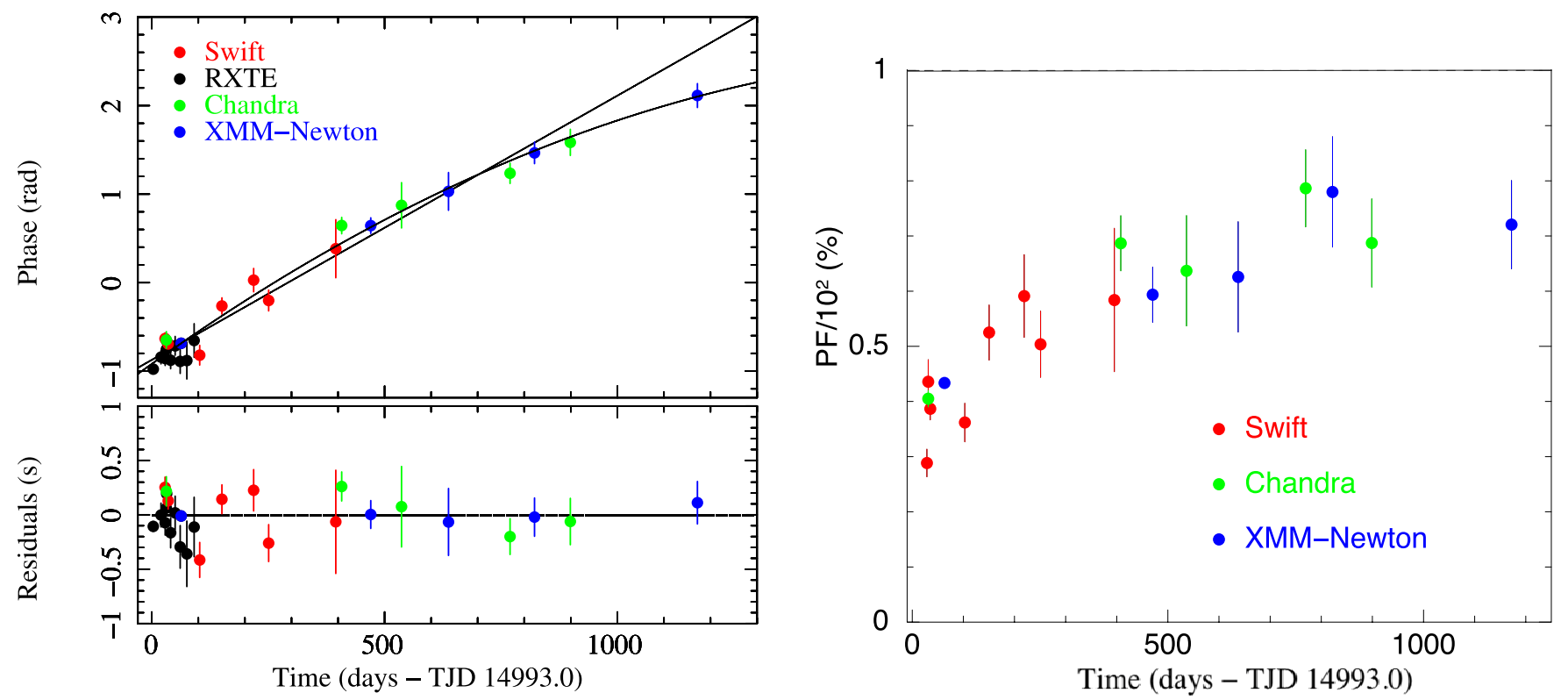

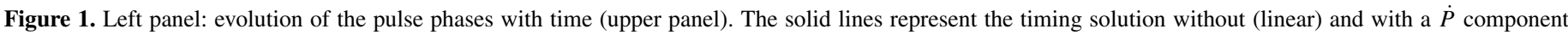

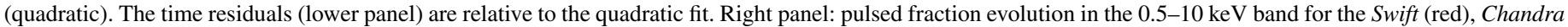
(green), and XMM-Newton (blue) observations.

(A color version of this figure is available in the online journal.)

cross-sections. We restricted our spectral modeling to the 0.7-10 keV energy band, excluding bad channels when needed. The Swift spectra were binned in order to have at least 30 counts per spectral bin. XMM-Newton spectra were grouped such to have at least 100, 50, and 40 photons per bin in the first three observations, respectively, and a minimum of 30 counts in the subsequent observations. On the other hand, all Chandra spectra have at least 25 counts per bin.

We started the spectral analysis by fitting all the spectra together (see Table 1) with a single component model: an absorbed blackbody or a power-law model. While the former gave a good fit, a single power law could not reproduce all the spectra at the same time. Fixing the absorption value to be the same for all spectra, for a single blackbody model (phabs*bbodyrad) we find an acceptable fit with $N_{\mathrm{H}}=$ $(1.15 \pm 0.06) \times 10^{21} \mathrm{~cm}^{-2}$ and $\chi_{v}^{2}=1.19$ (940 dof; errors on the spectral parameters are all reported at $90 \%$ confidence level). However, not unexpectedly, the best collected spectrum (the first XMM-Newton observation on 2009-08-12; see Table 1) gave bad residuals at lower and higher energies (see Figure 2, left panel). We tried to model this observation alone, and indeed a single absorbed blackbody or power-law components were not reproducing this spectrum properly $\left(\chi_{v}^{2}>2\right)$. We then used a composite model. Good fits were found using both an absorbed blackbody plus a power law (phabs*(bbodyrad + power); $N_{\mathrm{H}}=(6.32 \pm 0.04) \times 10^{21} \mathrm{~cm}^{-2}, k T=0.91 \pm 0.07 \mathrm{keV}$, $\Gamma=2.82 \pm 0.16$, and $\chi_{v}^{2}=0.97$ for $392 \mathrm{dof}$ ) and an absorbed resonant cyclotron scattering model (RCS: Rea et al. 2007, 2008, or NTZ: Zane et al. 2009). Two blackbodies were also producing acceptable reduced chi-square values $\left(\chi_{v}^{2}=1.01\right.$ for 392 dof) but with worse residuals at higher energies (this is compatible with what was found in Esposito et al. 2011 and Turolla et al. 2011). The parameters we found for the resonant cyclotron scattering models are $N_{\mathrm{H}}=(1.9 \pm 0.3) \times 10^{21} \mathrm{~cm}^{-2}$, $\tau=8.8 \pm 1.2, \beta=0.21 \pm 0.08$, and $k T=0.63 \pm 0.11 \mathrm{keV}$ $\left(\chi_{v}^{2}=1.08\right.$ for $\left.392 \mathrm{dof}\right)$ for the RCS model; and $N_{\mathrm{H}}=$ $(1.8 \pm 0.2) \times 10^{21} \mathrm{~cm}^{-2}, \Delta \phi=1.9 \pm 1.0, \beta_{\text {bulk }}=0.13 \pm 0.05$, and $k T=0.88 \pm 0.07 \mathrm{keV}\left(\chi_{v}^{2}=1.11\right.$ for $\left.392 \mathrm{dof}\right)$ for the NTZ model.

We then continued our spectral modeling of all spectra together by adding a further component only for this observation (adding a further component for all spectra did not significantly change the goodness of the fit; $\chi_{v}^{2}=1.13$ (938 dof); see Figure 2, middle and right panels). In Table 1 we report the values of the single absorbed blackbody model (see also Figure 2, left panel, and Figures 3 and 4), since when fitting all the spectra by using a composite model only for the first $X M M$-Newton observation, we find no change in the parameters of the other spectra with respect to the single blackbody fit. However, although a blackbody plus power-law model gives a good fit when fitting the first XMM-Newton observation alone, it is not so when fitting all data together. This is because the powerlaw component produces an unrealistic $N_{\mathrm{H}}$ increase, which does not match the value required by all the other observations modeled by a single blackbody. We then use one of the resonant cyclotron scattering models, the RCS model, for the joint fit as an empirical model for the first XMM-Newton observation. ${ }^{18}$

In addition to the joint spectral modeling, we also fitted all the spectra individually. Beside the first XMM-Newton observation discussed above, the last XMM-Newton observation, when fitted alone with a single blackbody model, did not give a good chisquare $\left(\chi_{v}^{2}=2.2\right.$ for $\left.16 \mathrm{dof}\right)$. Given the low number of counts in the spectrum of this observation ( $\sim 400$ background-subtracted counts), this deviation from the blackbody model had only a marginal effect on the joint fit. A better fit was found adding a second blackbody $\left(\chi_{v}^{2}=1.2\right.$ for 14 dof) or (with a slightly worse chi-square) a power-law component $\left(\chi_{v}^{2}=1.5\right.$ for 14 dof $)$. However, given the reduced number of counts collected in this

\footnotetext{
18 Note that both the RCS and NTZ models are built for higher surface dipolar fields, hence the fact that they provide a very good fit to the data is probably just an indication of the presence of some magnetospheric distortion. However, no real physical information can be derived from the resulting magnetospheric parameters. For the purpose of this work, we are mainly interested in the surface thermal cooling of the source.
} 

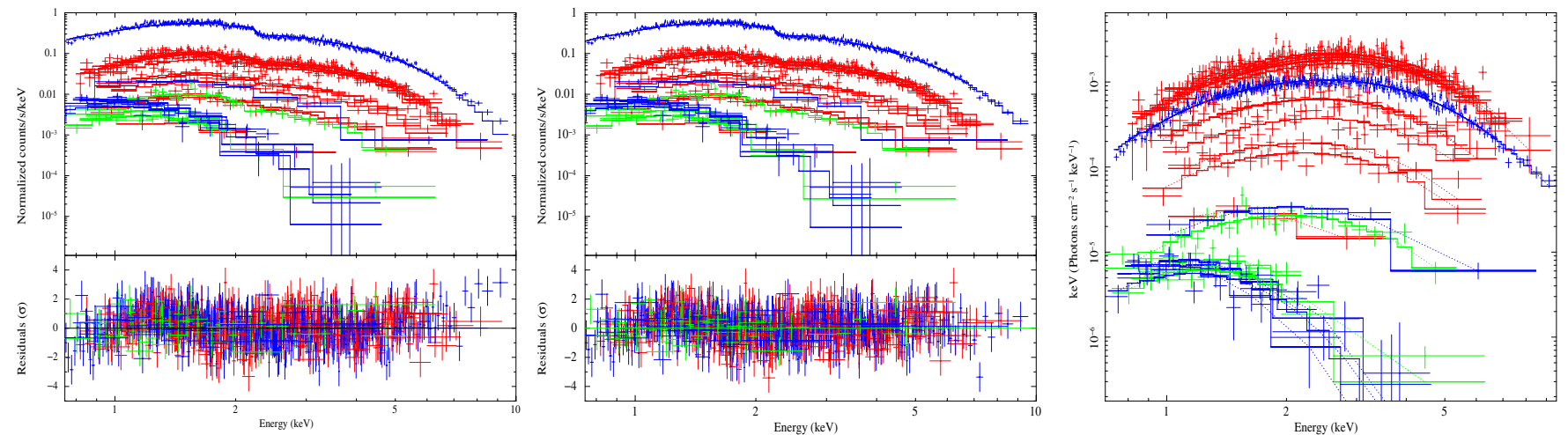

Figure 2. Spectral modeling of all observations listed in Table 1. Left and middle panels: spectra and residuals for all observations (Swift (red), Chandra (green), and $X M M$-Newton (blue)) fitted simultaneously with a single blackbody model (left) and using an RCS model only for the first XMM-Newton observation (center). Right panel: unfolded spectrum relative to the modeling shown in the central panel.

(A color version of this figure is available in the online journal.)

observation, a detailed modeling of the quiescent spectrum of SGR $0418+5729$ will be possible only when more data is accumulated.

We also tried to (1) model all the spectra with two blackbodies leaving one of the blackbodies with a fixed area mimicking the whole surface emission, and (2) fix one blackbody to the value observed in the last observation (see Table 1) and leave the second blackbody free to vary. In neither of those two cases could we find any improvement in the modeling of the data. Note that although a joint two blackbody model can fit the first few observations (Turolla et al. 2011), this is no longer the case when modeling together all the data collected in the entire 1200 day time span.

\subsection{X-Ray Timing Analysis}

All the Chandra and XMM-Newton event files collected between 2010 November and 2012 August were used in order to extend the coherent timing solution we derived in Rea et al. (2010): $P=9.07838827(4)$ s $90 \%$ c.l., and $3 \sigma$ first period derivative upper limit of $|\dot{P}|<6.0 \times 10^{-15} \mathrm{~s} \mathrm{~s}^{-1}$ at epoch 54993.0 MJD. Photon arrival times were corrected to the barycenter of the solar system. ${ }^{19}$ Timing analysis was carried out by means of a phase-fitting technique (details on this technique are given in Dall'Osso et al. 2003; see also Esposito et al. 2010 for further details on this source). Given the intrinsic variability of the pulse shape as a function of time (see Figure 6), we inferred the phase of the modulation by fitting the average pulse shape of each observation with a number of harmonics, the exact number of which is variable and determined by requesting that the inclusion of any higher harmonic is statistically significant (by means of an $F$ test). All data reported in Table 1 were folded using a reference period $9.07838880562798 \mathrm{~s}$ at epoch 54993 MJD, and fitted with one or more harmonics. In Figure 1 we plot the phases at which the fundamental sine function is equal to zero in its ascending part (positive derivative).

The fit of the resulting pulse phases with a linear component gives a reduced $\chi_{r}^{2} \sim 3.2$ for 26 degrees of freedom (dof hereafter). The inclusion of a quadratic term, corresponding to a first period derivative component, was found to be significant at a confidence level of $3.5 \sigma$ (by means of an $F$ test). The resulting best-fit solution corresponds to $P=9.07838822(5) \mathrm{s}$

\footnotetext{
19 We have corrected the arrival times of the last XMM-Newton observation for the 2012 June 30 leap second (see http://xmm.esa.int/sas/current/watchout/ 12.0.0/leapsec_2012.shtml for further details).
}

( $1 \sigma$ c.l., two parameters of interest; epoch 54993.0 MJD) and $\dot{P}=4(1) \times 10^{-15} \mathrm{~s} \mathrm{~s}^{-1}$ with a reduced $\chi_{r}^{2} \sim 2.1$ (for 25 dof; see also Figure 1). The new timing solution implies an rms variability of only $0.2 \mathrm{~s}$. As depicted above, the time evolution of the phase can be described by a relation of the form $\phi=\phi_{0}+2 \pi\left(t-t_{0}\right) / P-\pi\left(t-t_{0}\right) 2 \dot{P} / P^{2}$.

To further assess the significance of the quadratic component, reflecting the period derivative, we performed detailed Monte Carlo (MC) simulations assuming as the null model a simple linear relation (see Protassov et al. 2002 for further details). By running $10^{5} \mathrm{MC}$ simulations we verified that the quadratic component is significant at $>99.96 \%$ confidence level, which is in very good agreement with what was estimated by means of the $F$ test. We notice that examining the simulated data with different sampling distributions does not significantly change the results. Furthermore, we performed the same MC simulations using the whole set of observations but without considering the last XMM-Newton observation. We find a chance probability for the addition of a quadratic component of $0.65 \%(<3 \sigma)$. Given the strong influence of the last of our observations in the determination of the period derivative, we will perform further $\mathrm{X}$-ray observations in the next few years in order to increase the significance of the current $\dot{P}$ measurement.

Using this $\dot{P}$ measurement, we infer a surface dipolar magnetic field strength of $B_{\text {dip }}=(6 \pm 2) \times 10^{12} \mathrm{G}$, calculated at the neutron star equator. This value is fully consistent with the $3 \sigma$ upper limit reported in Rea et al. (2010). We also estimate a characteristic age of $\tau_{\mathrm{c}} \simeq P / 2 \dot{P} \sim 35 \mathrm{Myr}$, and a rotational power of $\dot{E} \simeq 3.9 \times 10^{46} \dot{P} / P^{3} \sim 2 \times 10^{29} \mathrm{erg} \mathrm{s}^{-1}$.

Based on the above phase coherent timing solution, we also studied the pulse shape and pulsed fraction evolution. Figure 1 shows the pulsed fraction evolution as a function of time. There is an evident increase starting soon after the burst detection with a recovery toward an asymptotic quiescent value which appears to be at about the $70 \%-80 \%$ level.

In Figures 1, 5, and 6 we study in detail the shape of the pulse profile as it evolves in time and in energy. By looking at the profile shapes of all X-ray observations performed so far, the source appears to be switching among a triple/double/single peak shape during the early outburst phases, with no clear trend in time (Figure 1). The pulse profile stabilizes to a single peak about three months after the outburst onset. However, studying in detail the first and the last XMM-Newton observations, a few key pieces of information can be extracted: (1) at lower energies $(<1 \mathrm{keV})$, the pulse profile is mainly single peaked, 

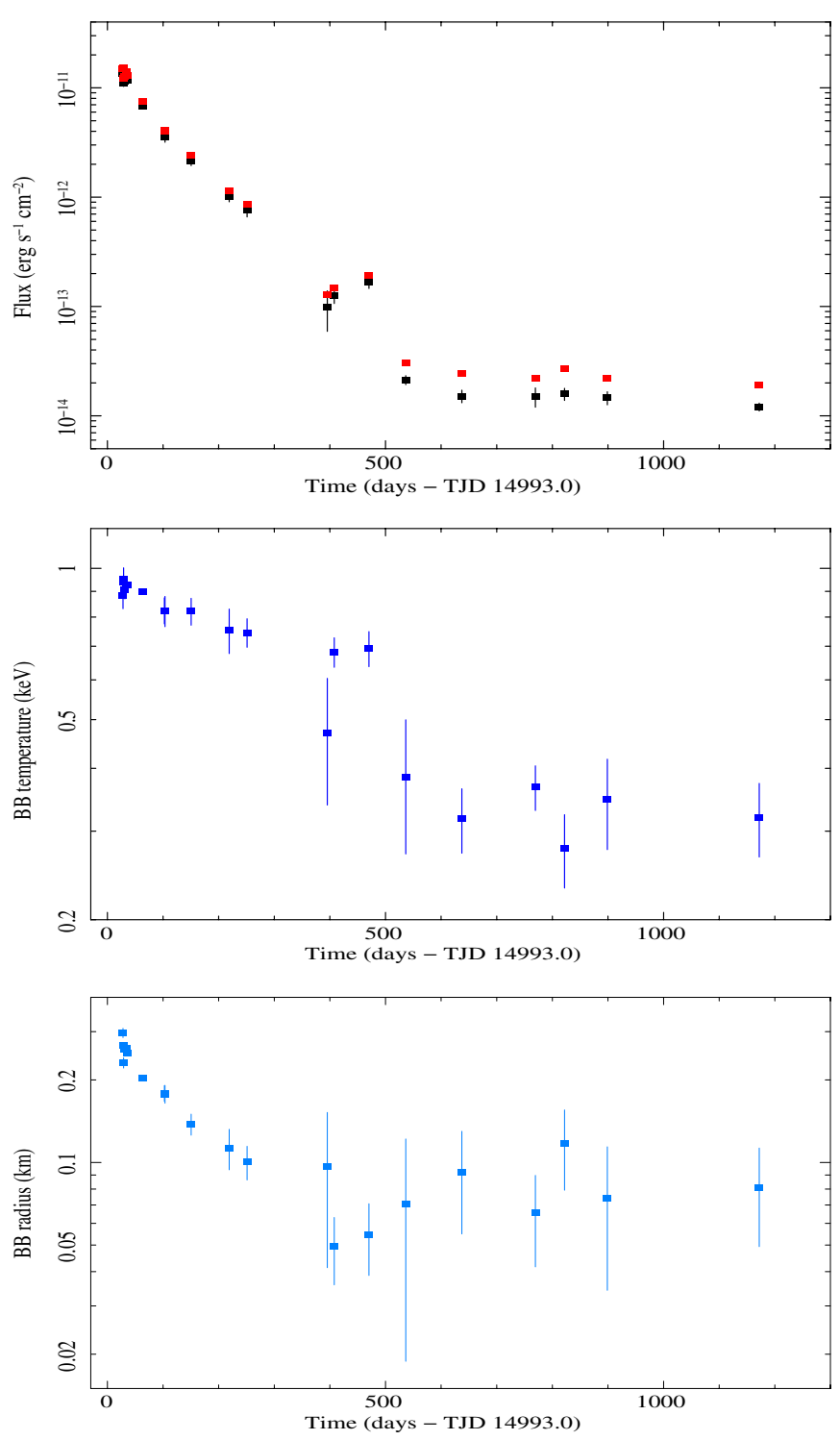

Figure 3. Spectral evolution with time. Top panel: flux evolution for the absorbed 0.5-10 keV flux (black), and for the bolometric unabsorbed flux (red). Middle and bottom panels: evolution of the blackbody temperature and radius, calculated at infinity (the latter assuming a $2 \mathrm{kpc}$ distance).

(A color version of this figure is available in the online journal.)

while a second, and possibly also a third peak, appears at higher energies (see Figure 5); (2) the main component of the pulse profile continues to be at the same phase over the whole outburst decay (see Figure 7).

\subsection{Pulse Phase Spectroscopy}

We performed a pulse phase spectroscopy of the first $X M M-N e w t o n$ observation. A clear pulse phase dependence of the spectrum is already observed by simply looking at the pulse profile changes as a function of the energy (see Figure 5). In order to quantify the spectral variability as a function of the rotational phase, we performed a pulse phase spectroscopy extracting the spectra from phases $0-0.4,0.4-0.6$, and $0.6-1$. These phase intervals were chosen by looking at Figure 5 in order to isolate the dip in the $1-4 \mathrm{keV}$ pulse profiles at phase $\sim 0.55$. In Table 2 we report the results of our modeling. The phase-averaged spectrum is not well fit by either a single blackbody nor a power law. We then used an absorbed blackbody

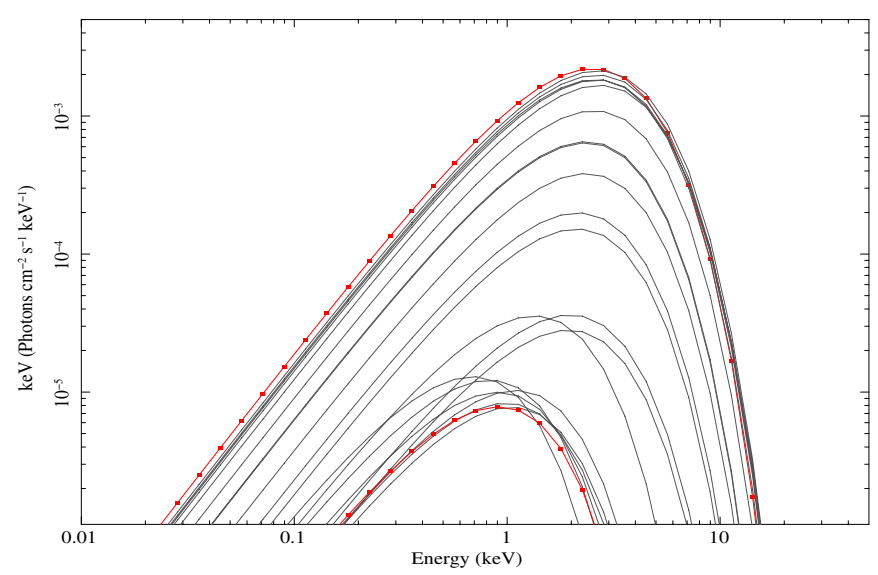

Figure 4. Fitted blackbody models (see Table 1), with the first and last observations labeled as red squares (see Figure 3 and text for details).

(A color version of this figure is available in the online journal.)

Table 2

Pulse Phase Spectroscopy of the First XMM-Newton Observation of SGR $0418+5729$

\begin{tabular}{lccr}
\hline \hline Phase & Counts s $^{-1}$ & Flux $^{\mathrm{a}}$ & Photon Index $^{\mathrm{b}}$ \\
\hline $0.0-0.4$ & $1.46 \pm 0.01$ & $7.5 \pm 0.1(1.71 \pm 0.02)$ & $2.86 \pm 0.15$ \\
$0.4-0.6$ & $1.00 \pm 0.01$ & $5.5 \pm 0.1(1.20 \pm 0.02)$ & $4.08 \pm 0.44$ \\
$0.6-1.0$ & $1.26 \pm 0.01$ & $6.6 \pm 0.1(1.47 \pm 0.02)$ & $3.11 \pm 0.21$ \\
\hline
\end{tabular}

Notes.

${ }^{a}$ Absorbed flux in the $0.5-10 \mathrm{keV}$ energy range, and in units of $10^{-12} \mathrm{erg} \mathrm{s}^{-1}$ $\left(10^{-3}\right.$ photons $\left.\mathrm{cm}^{-2} \mathrm{~s}^{-1}\right)$. See also Section 3.3.

${ }^{\mathrm{b}}$ Fitted model is phabs* (bbodyrad+power); $N_{\mathrm{H}}=(6.9 \pm 0.5) \times 10^{21} \mathrm{~cm}^{-2}$, $k T_{\mathrm{BB}}=0.91 \pm 0.01 \mathrm{keV}, \mathrm{BB}$ norm $=0.82 \pm 0.05$, and $\chi_{v}^{2}=0.96$ (for $680 \mathrm{dof}$ ).

plus power-law modeling (note that the RCS and NTZ models are not suited for phase resolved analysis since they are intrinsically phase-average), using for the photoelectric absorption model the same cross-section and abundances as for the phaseaverage spectrum (see Section 3.1). The blackbody temperature and radius were consistent in all three spectra, hence we fixed them to be the same for all spectra $\left(k T_{\mathrm{BB}}=0.91 \pm 0.01 \mathrm{keV}\right.$, $\mathrm{BB}$ norm $=0.82 \pm 0.05$ ), while a variability $>3 \sigma$ has been observed in the photon index (it changed from about 2.9 to 4.1 between the spectra of the first peak and the dip).

However, from Figure 7 it is clear that the main difference in the spectra is at lower energies. In particular, above $5 \mathrm{keV}$ the three spectra are very similar, while the $0.4-0.6$ phase-resolved spectrum seems to have less counts than the other below such energy.

\section{GREEN BANK TELESCOPE RADIO OBSERVATIONS}

We observed SGR 0418+5729 using the $101 \mathrm{~m}$ Green Bank Telescope (GBT) on 2012 October 4, during the return to quiescence. Data were acquired with the Green Bank Ultimate Pulsar Processing Instrument (GUPPI; DuPlain et al. 2008) at a central frequency of $2.0 \mathrm{GHz}$ (with a bandwidth of $800 \mathrm{MHz}$, integration time of $\sim 5400 \mathrm{~s}$ and sampling time of $655 \mu \mathrm{s}$ ) and $820 \mathrm{MHz}$ (with a bandwidth of $200 \mathrm{MHz}$, integration time of $\sim 5600 \mathrm{~s}$, and sampling time of $655 \mu \mathrm{s}$ ). To minimize the dispersive effects of the interstellar medium, the bandwidths were split into 2048 and 512 channels, respectively. The working of the system was checked looking at the pulsar PSR B0450+55. A mask was first applied to the full resolution data for reducing the effects of impulsive RFI and of bad channels. Then the 

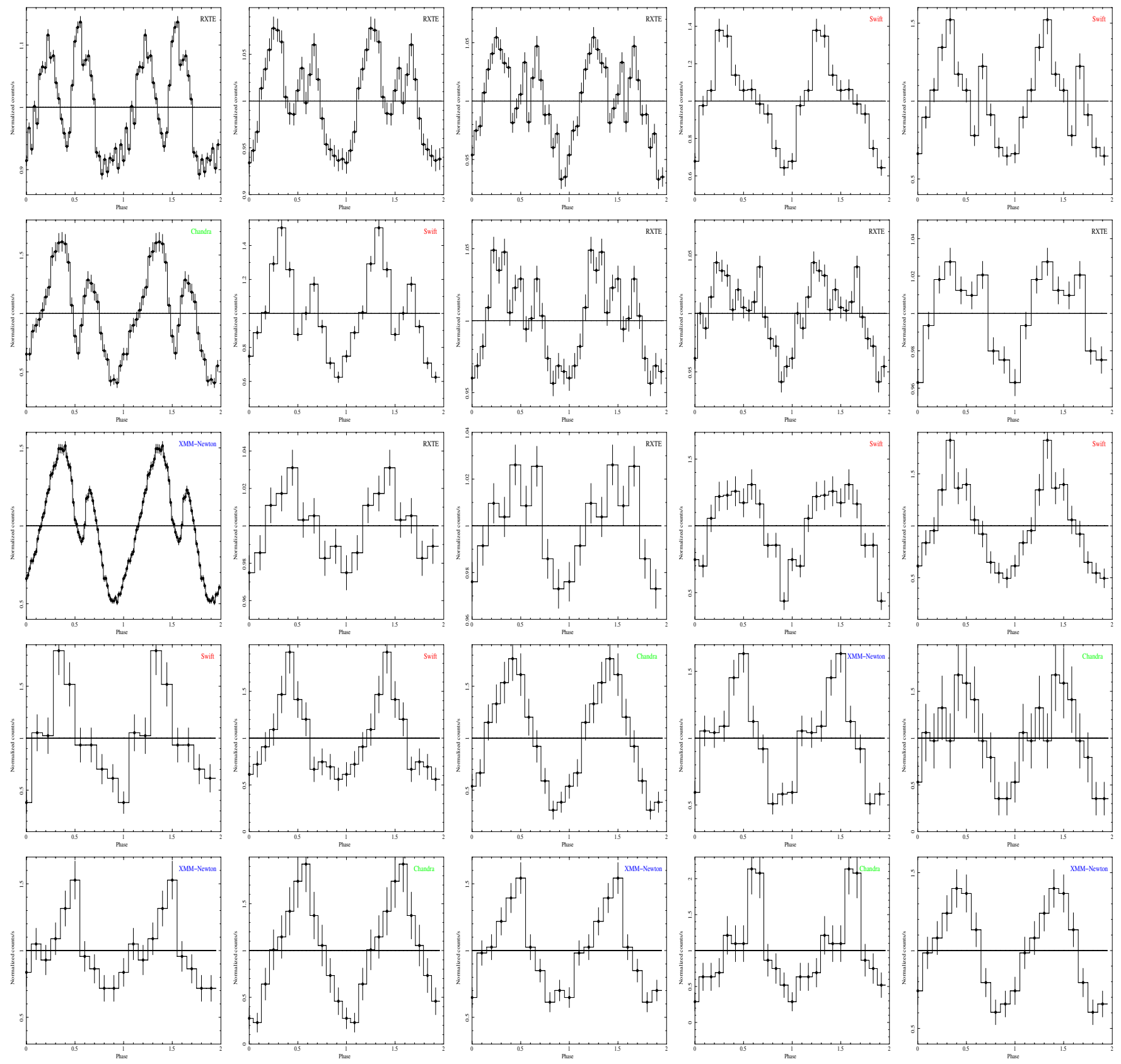

Figure 5. Pulse profile (normalized counts $\mathrm{s}^{-1}$ vs. phase) as a function of energy, relative to the first XMM-Newton observation (see Table 1).

(A color version of this figure is available in the online journal.)

cleaned data were downsampled a factor two in sampling time, matching the frequency resolution in order to have a maximum dispersion smearing of order $1.3 \mathrm{~ms}$ in each channel for a pulsar with dispersion measure $(\mathrm{DM}) \sim 100 \mathrm{pc} \mathrm{cm}^{-3}$.

The ephemerides acquired from the X-ray observations (see Section 3.2), were used to fold the resulting data in $\sim 3$ minutes long sub-integrations at the known magnetar period. We also folded the data at half, one-third, and a quarter of the nominal period in order to detect putative higher harmonics components of the intrinsic signal, in case the latter were deeply contaminated by interference (RFI). Folding was done using dspsr (van Straten \& Bailes 2010). The sub-integrations and the frequency channels, cleaned from RFI, were then searched around the pulsar period $P$ and over a wide range of DM values (from 0 to $1000 \mathrm{pc} \mathrm{cm}^{-3}$ ) to find the $P-\mathrm{DM}$ combination maximizing the signal-to-noise ratio. No dispersed signal was found in the data down to a signal-to-noise limit of 10 in both data sets. Given the parameters of the antenna and of the receivers, ${ }^{20}$ and assuming a pulsar with a duty cycle of $10 \%$, that translates to flux densities of $\sim 0.02 \mathrm{mJy}$ and $\sim 0.05 \mathrm{mJy}$, for the $2 \mathrm{GHz}$ and $820 \mathrm{MHz}$ observations, respectively. Data were also blindly searched for a periodic signal in the Fourier domain, and for single de-dispersed pulses (within a DM range from 0 to $\left.200 \mathrm{pc} \mathrm{cm}^{-3}\right)$. No signal was found in either the Fourier domain (down to a spectral signal-to-noise ratio of 4) or in the single pulse searches (down to a signal-to-noise ratio of 5 for the individual pulses).

No previous search for pulsed radio emission had been performed at $2 \mathrm{GHz}$, whereas the flux density value at $820 \mathrm{MHz}$

\footnotetext{
${ }^{20}$ http://www.gb.nrao.edu/gbtprops/man/GBTpg.pdf
} 
improves by $\sim 15 \%$ the limit of the observation at $820 \mathrm{MHz}$ performed on 2009 July 19 (Lorimer et al. 2009, ATel 2096), when the source was in the phase of outburst. Assuming a typical pulsar spectral index of 1.7 , a typical duty cycle $\sim 10 \%$ and a distance of $2 \mathrm{kpc}$ (van der Horst et al. 2010), the observations at $820 \mathrm{MHz}$ sampled more than $97 \%$ of the luminosity distribution of the population of known ordinary pulsars with rotational period longer than $100 \mathrm{~ms}$, as derived from the ATNF pulsar catalog. ${ }^{21}$

\section{PLATEAU DE BURE MM OBSERVATIONS}

SGR 0418+5729 was observed with the Plateau de Bure Interferometer $(\mathrm{PdBI})$ at $1.8 \mathrm{~mm}(166.50 \mathrm{GHz})$ in the $\mathrm{D}$ configuration between 2011 June and July (June 27, and July 9, 10, 15, and 16). This configuration provides baselines between 22.1 and $95.6 \mathrm{~m}$. The phase center of the observations was $04: 18: 33.867,+57: 32: 22.910$. The dominant track was July 15 (eight-hour track and excellent weather conditions). The system temperatures were typically in the 150 to $200 \mathrm{~K}$ range, and the averaged atmospheric precipitable water vapor was $2 \mathrm{~mm}$. The gain calibration was performed observing the quasars B0552+398 and J0512+294. After calibration, the phase rms was $20^{\circ}-60^{\circ}$. The bandpass calibrator used was B $0851+202$. The adopted flux density for the flux calibrator 3C273 was $16.57 \mathrm{Jy}$. Calibration and imaging were performed using the standard procedures in the CLIC and MAPPING packages of the GILDAS ${ }^{22}$ software. The resulting final map, obtained combining all the data, yields a synthesized beam size of $3^{\prime \prime} .95 \times 3$ ". 16 with a position angle of P.A. $=0.0$. The rms noise achieved using the full $3.6 \mathrm{GHz}$ provided by the WideX correlator is $60 \mu \mathrm{Jy}$ beam $^{-1}$. The primary beam of the PdBI at $166.50 \mathrm{GHz}$ is $30^{\prime \prime} .3$.

We did not detect continuum emission within the PdBI primary beam toward SGR $0418+5729$, and obtained an upper limit of $0.24 \mathrm{mJy}$ beam $^{-1}$ at a $4 \sigma$ level (see Figure 8 ). The only detected source is at R.A. $=04: 18: 30.077$, decl. $=$ 57:32:52.00, which corresponds to an offset of $\left(30^{\prime \prime} .5,29^{\prime \prime} .1\right)$ with respect to the phase center, or a total offset of 42 ". 1 . The flux density of this millimeter source is $0.34 \pm 0.06 \mathrm{mJy}$ (from a Gaussian fit in the $u v$ plane and without correcting for the primary beam response). In addition, we looked for possible "pulses" of emission at $1.8 \mathrm{~mm}$. In order to do that, we checked the calibrated amplitude versus time for the longest track (July 15). By averaging the visibilities in intervals of 1 minute, we found no hints of variable emission at an upper limit of roughly $\sim 10 \mathrm{mJy}$.

We also searched the NRAO Very Large Array Sky Survey at $21 \mathrm{~cm}$ and found no source within $15^{\prime}$ of the millimeter source (Condon et al. 1998; limiting brightness: $2.0 \mathrm{mJy}^{\text {beam }}{ }^{-1}$ ).

\section{WILLIAM HERSCHEL TELESCOPE OPTICAL OBSERVATIONS}

We acquired four $300 \mathrm{~s} r$-band images of the field containing SGR 0418+5729 on 2009 August 16, using the ACAM imager mounted at the $4.2 \mathrm{~m}$ William Herschel Telescope (WHT) on La Palma. The average seeing was $1^{\prime \prime}$ and airmass 1.26. Observations of a nearby field containing Sloan Digital Sky Survey calibrated stars were obtained for the absolute photometric calibration, while astrometry was performed against Two Micron

\footnotetext{
21 http://www.atnf.csiro.au/research/pulsar/psrcat/

22 The GILDAS data reduction package is available at http://www.iram.fr/IRAMFR/GILDAS.
}

All Sky Survey sources, resulting in an accuracy of $\sim 0^{\prime} .1$ on both R.A. and decl.

No source was detected within the $95 \%$ confidence down to a limit of $r=24$. PSF photometry reveals that the nearest object is detected at a magnitude $r=22.7 \pm 0.1$ and center coordinates R.A. $=04: 18: 34.0$, decl. $=57: 32: 23.5$. This source is consistent with the near-infrared source reported by Wachter et al. (2009), but its distance from the SGR 0418+5729 position $\left(\sim 1^{\prime \prime}\right.$. 4 ), makes the association with the magnetar rather unlikely.

\section{FERMI-LAT GAMMA-RAY OBSERVATIONS}

We used data from the Large Area Telescope (LAT) on board Fermi (Atwood et al. 2009) from 2008 August 4 until 2012 October 24. The Fermi SCIENCE TOOLS SC09-28-00 package was used to analyze the "source" event class data. We selected events within a circular region of interest (ROI) of $10^{\circ}$ radius centered on the position of SGR $0418+5729$, and in the energy range $100 \mathrm{MeV}-100 \mathrm{GeV}$. The good time intervals are defined so that the ROI does not fall below the gamma-ray-bright Earth limb (defined at $100^{\circ}$ from the zenith angle), and the source is always inside the LAT field of view, namely in a cone angle of $66^{\circ}$. The "P7SOURCE_V6" instrument response functions (IRFs) are applied in the analysis.

The likelihood analysis of SGR $0418+5729$ was performed by means of the binned maximum-likelihood method (Mattox et al. 1996), using the official tool gtlike released by the Fermi-LAT collaboration. The spectral-spatial model created for the likelihood analysis includes the Galactic, and the isotropic diffuse emission models, as well as all the 2FGL sources within a radius of $15^{\circ}$ from SGR $0418+5729$. Since there is no 2 FGL source that is positionally associated to the magnetar, we added in the spectra-spatial model a point-like source modeled with a simple power-law with the coordinates of SGR $0418+5729$. The 2FGL sources within $3^{\circ}$ of SGR $0418+5729$ (3 sources) are modeled with the flux parameter allowed to vary, while the others 34 sources had all their parameters fixed to the value from the 2FGL catalog. Figure 8 shows the diffuse subtracted TS map of the $7^{\circ} \times 7^{\circ}$ region centered on SGR $0418+5729$. It was obtained associating to each pixel (of size $0.1 \times 0.1$ ) the TS value calculated assuming a point-like testing source in its center. Diffuse subtracted TS map means that the spectralspatial model for the null hypothesis includes only the Galactic and isotropic emission models, so that the point-like sources should be visible in the map.

As no significant gamma-ray counterpart to SGR 0418+5729 is identified, 95\% flux upper limit is derived using the Bayesian method developed by Abdo et al. (2010). The 95\% flux upper limit for $E>100 \mathrm{MeV}$ is $F<1.3 \times 10^{-8}$ photons $\mathrm{cm}^{-2} \mathrm{~s}^{-1}$, including systematics.

The non-detection of SGR $0418+5729$ at energies $>100 \mathrm{MeV}$ is not surprising, given a similar non-detection of all other known magnetars (Abdo et al. 2010).

\section{DISCUSSION}

We have presented here a detailed X-ray study of the outburst of the low magnetic field soft gamma repeater SGR 0418+5729. The long-term monitoring we performed over 1200 days allowed us to measure the period derivative of this pulsar $(\dot{P}=4(1) \times$ $10^{-15} \mathrm{~s} \mathrm{~s}^{-1}$ ) with a $3.5 \sigma$ significance (Figure 1 ). This yields an estimate of its dipolar magnetic field of $B_{\text {dip }} \sim 6 \times 10^{12} \mathrm{G}$, and confirms this object as the magnetar with the lowest dipolar magnetic field ever discovered. 
Assuming that SGR $0418+5729$ attained its quiescent state in the last few observations, the $\mathrm{X}$-ray quiescent emission appears to be dominated by a very small spot at $k T \sim 0.3 \mathrm{keV}$ and of radius $\sim 0.16 \mathrm{~km}$ (assuming a $2 \mathrm{kpc}$ distance), which corresponds to a cap of semi-aperture $\sim 1^{\circ}-2^{\circ}$, similar to what observed in old radio pulsars. However, in the present case the rotation power $\left(\dot{E} \approx 10^{29} \mathrm{erg} \mathrm{s}^{-1}\right)$ is about two orders of magnitude smaller than the observed X-ray luminosity $\left(\approx 10^{31} \mathrm{erg} \mathrm{s}^{-1}\right)$, thus indicating a different origin for the emission, most likely magnetic. Actually, it is quite likely that most of the surface is at a much lower temperature and is therefore invisible at energies between $0.5-10 \mathrm{keV}$. This implies that the quiescent bolometric flux may be severely underestimated (see also below).

The study of the spectral evolution during the outburst shows the presence of a non-thermal component (probably magnetospherical) at the beginning of the outburst, which fades away after a few hundred days. On the other hand, the temperature of the small region responsible for the surface anisotropy fades from 0.9 to $0.3 \mathrm{keV}$ within a timescale of a few years (see Table 1 and Figure 3).

The pulse profile evolution during the outburst decay shows some interesting features. As Figure 6 shows, there is an overall trend toward a simplification of the pulse, which starts with a complex, three-peaked shape and ends with a fairly sinusoidal pattern. Furthermore, the study of the pulse profiles as a function of energy (Figure 5) in the first outburst stages, shows a great variability too. A large dip in an otherwise rather sinusoidal profile is observed at energies between $1-4 \mathrm{keV}$.

The large pulsed fraction of $40 \%-70 \%$ (Figure 1), the evidence of nearly phase aligned spots responsible for the $0.9 \mathrm{keV}$ thermal emission in the early outburst phases, as well as the $0.3 \mathrm{keV}$ emission at late times, disfavor the presence of two spots at different temperatures, while favoring the presence of a single tiny spot cooling down (from 0.9 to $0.3 \mathrm{keV}$ ) and reducing its size (from 0.21 to $0.16 \mathrm{~km}$ ) during the return to quiescence. This means that the multi-peaked pulse profile is probably due to anisotropies in the magnetospheric electrons distribution (on top of a non-isotropic surface thermal emission).

\subsection{SGR 0418+5729 as an Evolved Magnetar}

In Turolla et al. (2011) it was shown that the rotational properties of SGR $0418+5729$ can be reproduced if the source is an aged magnetar, which experienced substantial field decay but still retains a strong enough internal toroidal field. The most updated magneto-thermal evolutionary models discussed in Viganò et al. (2013; but see also Pons et al. 2009 and Aguilera et al. 2008), confirm this scenario. The evolution of an initial dipolar magnetic field of $B_{\text {dip }}^{0} \sim 1.5 \times 10^{14} \mathrm{G}$ (surface value at the pole) correctly provides the observed $P$ and $\dot{P}$ at an age of $\sim 550 \mathrm{kyr}$, which is probably the real age of this source.

Although different combinations of the initial components of the magnetic field are possible, in all the models the magnetic field must have been large in the past ( $\left.\gtrsim 10^{14} \mathrm{G}\right)$ to explain at the same time the long spin period, the bright $\mathrm{X}$-ray emission at this old age, and the flaring activity of the source. The characteristic age overestimates the real one by almost two orders of magnitude. In Figure 9, we show the evolution of period, period derivative, the source track in the $P-\dot{P}$ diagram, and the bolometric thermal luminosity. In this scenario we estimate that SGR 0418+5729's mean surface temperature should now be $\sim 0.05 \mathrm{keV}$, unfortunately undetectable by current

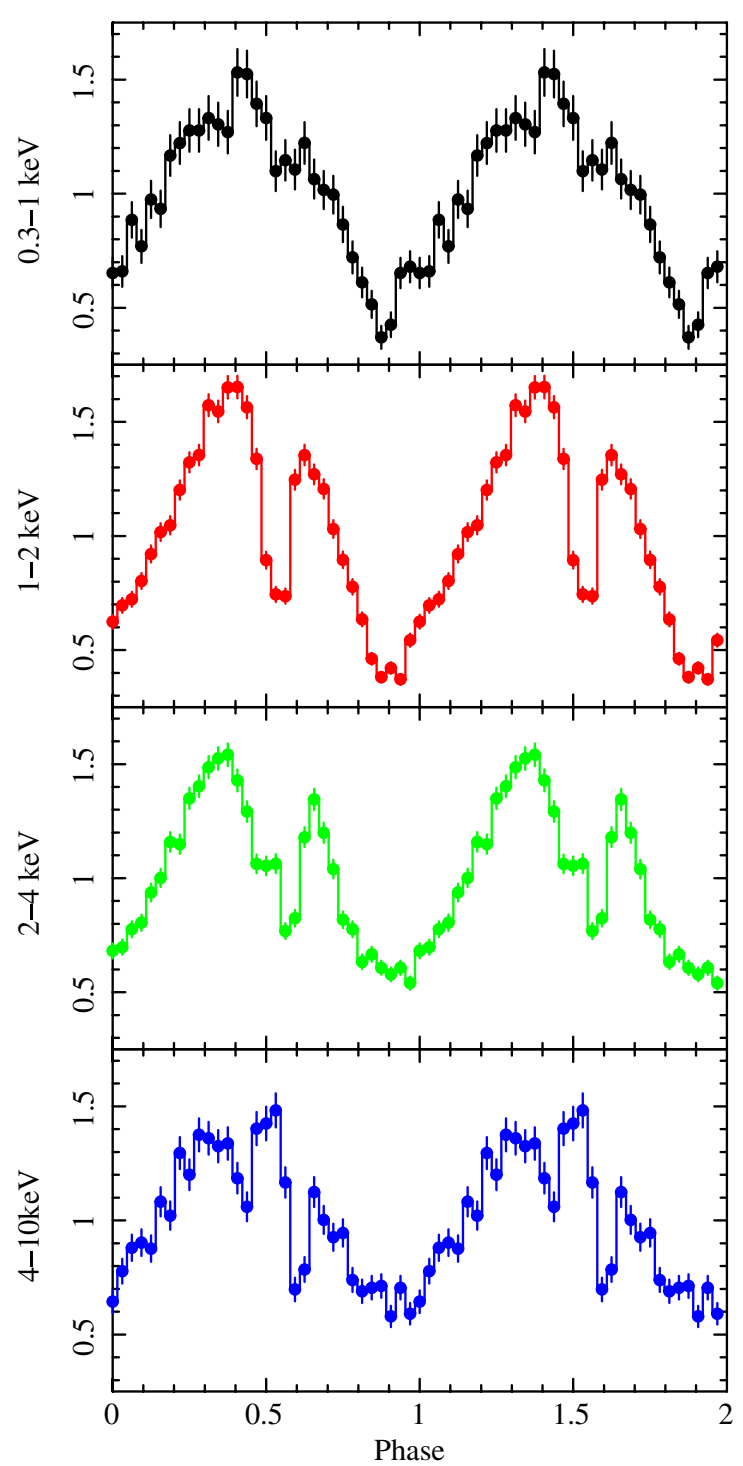

Figure 6. Pulse profiles evolution in the $2-10 \mathrm{keV}(R X T E)$ and $0.5-10 \mathrm{keV}$ (Chandra and XMM-Newton) energy ranges, for most of the observations reported in Table 1. Epoch increases from left to right, and top to bottom.

(A color version of this figure is available in the online journal.)

X-ray observations (which are observing only a hot tiny region on the star surface).

For the evolution of the timing properties, we assume the magneto-dipole braking formula given by Spitkovsky (2006): $I \Omega \dot{\Omega} \approx\left(B_{d}^{2} R^{6} \Omega^{4} / 4 c^{3}\right)\left(1+\sin \chi^{2}\right)$, where $R$ is the NS radius, $\chi$ is the angle between the rotational and the magnetic axis, $c$ is the speed of light, $\Omega=2 \pi / P$ is the angular velocity, and $I$ is the moment of inertia of the star.

An alternative possibility is that the neutron star was born with an external magnetic field close to the present one, but its large core poloidal field slowly diffuses out, i.e., by ambipolar diffusion (Soni 2012). Although from the timing properties alone it is hard to discriminate between a hidden strong crustal magnetic field, a hidden strong core field or an intrinsically low$B$ neutron star, the magnitude of the X-ray luminosity, and the spectral properties and light curves may be used to distinguish the different scenarios.

In particular, the low magnetic field scenario cannot explain the high luminosity, large pulsed fraction, and the flaring activity of the source. As a matter of fact, if there is little field decay and 

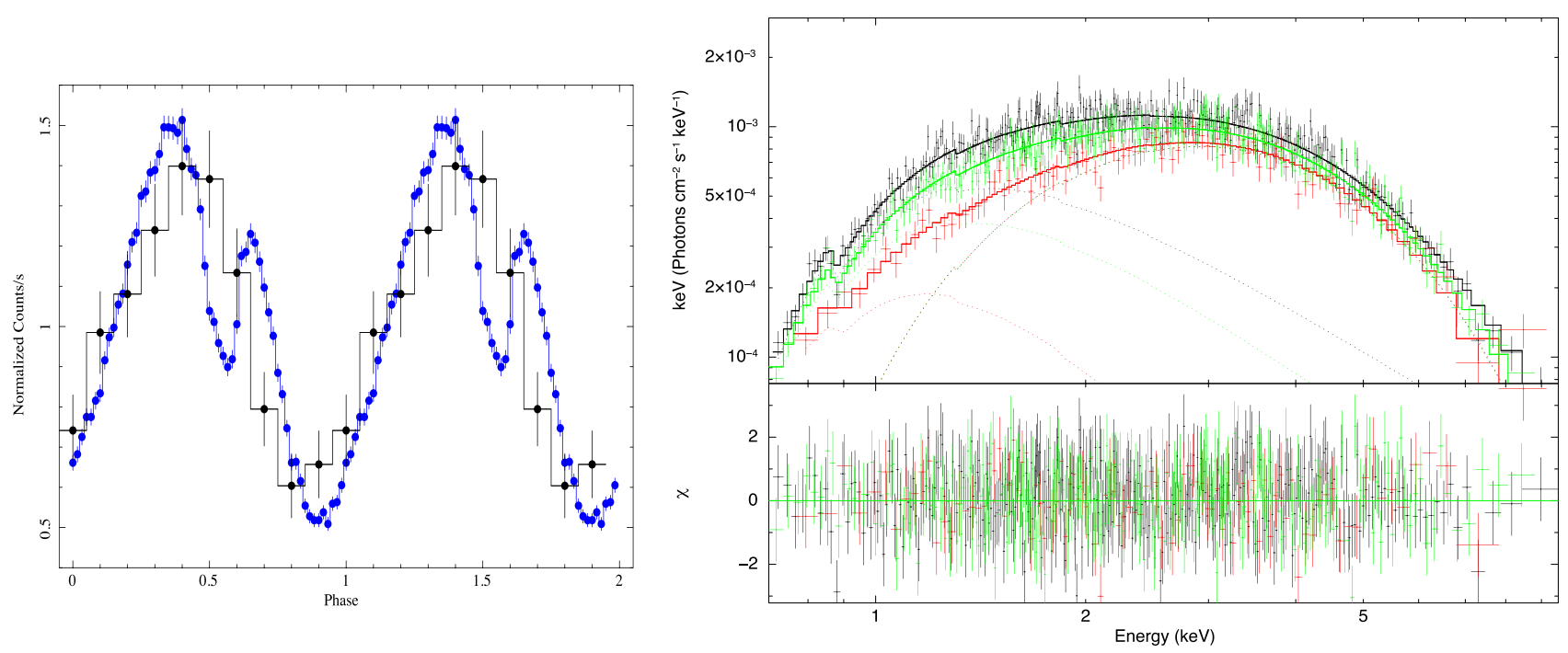

Figure 7. Left panel: pulse profile of the first (blue) and last (black) XMM-Newton observations in the $0.5-10 \mathrm{keV}$ energy band. Right panel: phase-resolved unfolded spectra for the first XMM-Newton observation. The spectra are relative to phases $0.0-0.4$ (black), $0.4-0.6$ (red), and 0.6-1 (green). The phase ranges are relative to the blue pulse profile in the left panel.

(A color version of this figure is available in the online journal.)
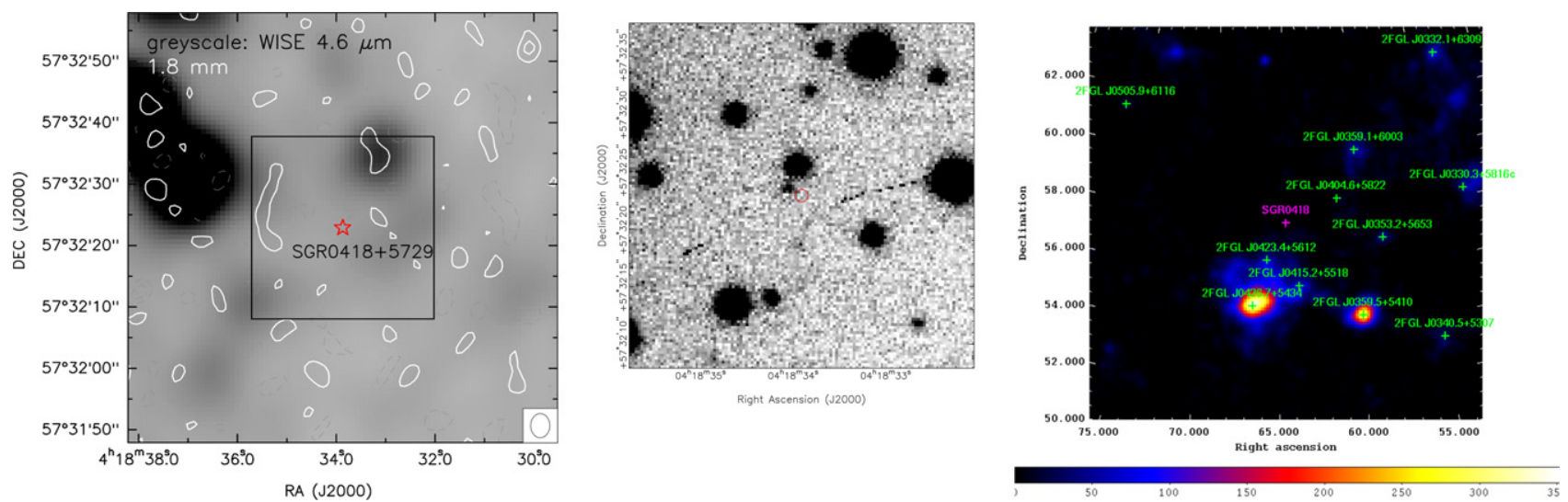

Figure 8. Left panel: contours of the $1.8 \mathrm{~mm}$ Plateau de Bure emission of the field of SGR $0418+5729$. Contours are $-4,-2$ (dashed gray), 2 , and 4 (white) times the rms noise of the map, $60 \mu \mathrm{Jy}$ beam $^{-1}$, and they are over-plotted on the Wide-field Infrared Survey Explorer image at $4.6 \mu \mathrm{m}$. The star symbol indicates the position of SGR $0418+5729$, and its size corresponds to its positional uncertainty $\left(\sim 1^{\prime \prime} .2\right.$ in diameter). The synthesized beam, of $3^{\prime \prime} .95 \times 33^{\prime \prime} 16$, at P.A. $=0^{\circ}$, is shown in the bottom right corner. The square indicates the field of view of the $r$-map acquired with the William Herschel Telescope (central panel). Central panel: William Herschel Telescope $r$-band field of SGR 0418+5729. Right panel: Fermi-LAT $(0.1-100 \mathrm{GeV})$. Diffuse subtracted TS map of the $7^{\circ} \times 7^{\circ}$ sky region centered on the magnetar position. The map is calculated for $E>300 \mathrm{MeV}$. The 2FGL sources are labeled in green, while SGR 0418+5729 in magenta.

(A color version of this figure is available in the online journal.)

the real age corresponds to the characteristic age (which in this scenario would be needed to reach the present period of $9 \mathrm{~s}$ ), no existing non-magnetic cooling model can account for an X-ray luminosity of $\approx 10^{31} \mathrm{erg} \mathrm{s}^{-1}$ at a characteristic age of $\approx 35 \mathrm{Myr}$.

The scenario in which a large core field diffuses out has the same problem: if the real age of the star is similar to its old characteristic age, no cooling model in the literature predicts such high quiescent luminosity. In addition, while the timescales used in Soni (2012) are correct for normal, nonsuperfluid nuclear matter, recent work (Glampedakis et al. 2011) shows that in the presence of superfluidity in the neutron star core, the timescales for ambipolar diffusion are many orders of magnitude longer, and therefore ambipolar diffusion does not play any role during the active age of the star.

\subsection{SGR 0418+5729 Outburst Rate}

An important question is whether a relatively low dipolar field is consistent with the star-quake model in which the primary cause of the outburst is an internal deposition of energy following a crust fracture. It is often overlooked that the magnetic stress needed to break the crust is strongly dependent on density (it is much easier to break the outer crust than the inner crust) and that the crust thickness grows as the temperature drops with age. In Figure 10 we show an estimate of the minimum magnetic field variation required to induce a fracture. As assumed in Perna \& Pons (2011), this estimate is obtained assuming that the crust moves through a series of equilibrium states in which its elastic stress balances the (time-dependent) magnetic stress. The deviation of the magnetic field with respect to the last unstressed configuration $\left(\delta B_{c}\right)$ may be large enough to break the crust when

$$
\delta B_{c} \approx\left(4 \pi \sigma_{b}^{\max }\right)^{1 / 2}
$$

where $\sigma_{b}^{\max }$ is the maximum stress that a neutron star crust can sustain. For young, relatively hot magnetars (crustal temperatures of $5 \times 10^{8} \mathrm{~K}$ ), only the inner crust is solid, and strong 

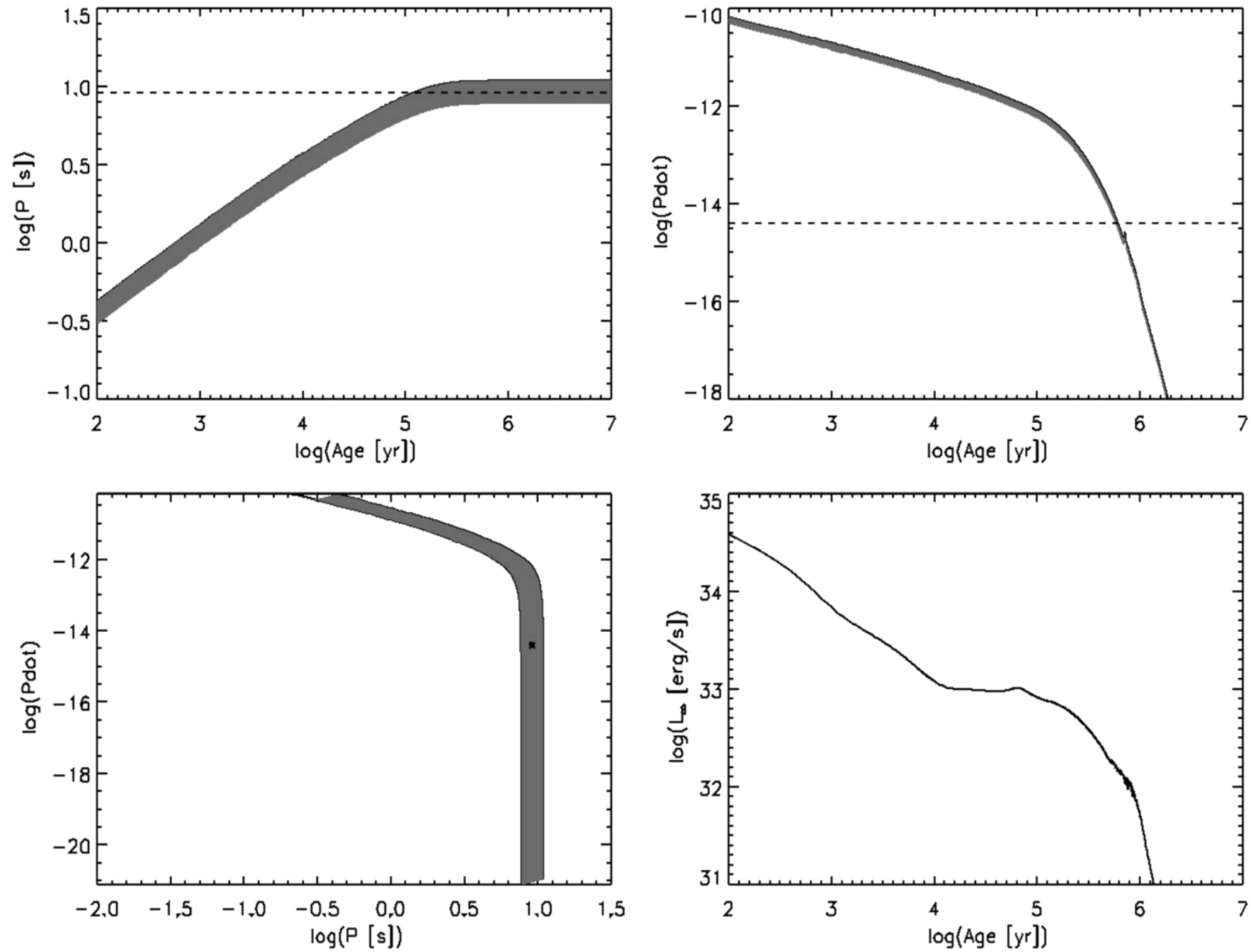

Figure 9. Magneto-thermal evolution of a neutron star with an initial poloidal field of $B_{\text {dip }}=1.5 \times 10^{14} \mathrm{G}$ : period (left-top), period derivative (right-top), evolution in the $P-\dot{P}$ diagram (left-bottom), and bolometric thermal luminosity (right-bottom). The gray band corresponds to the uncertainty of the angle-dependent spin-down formula.
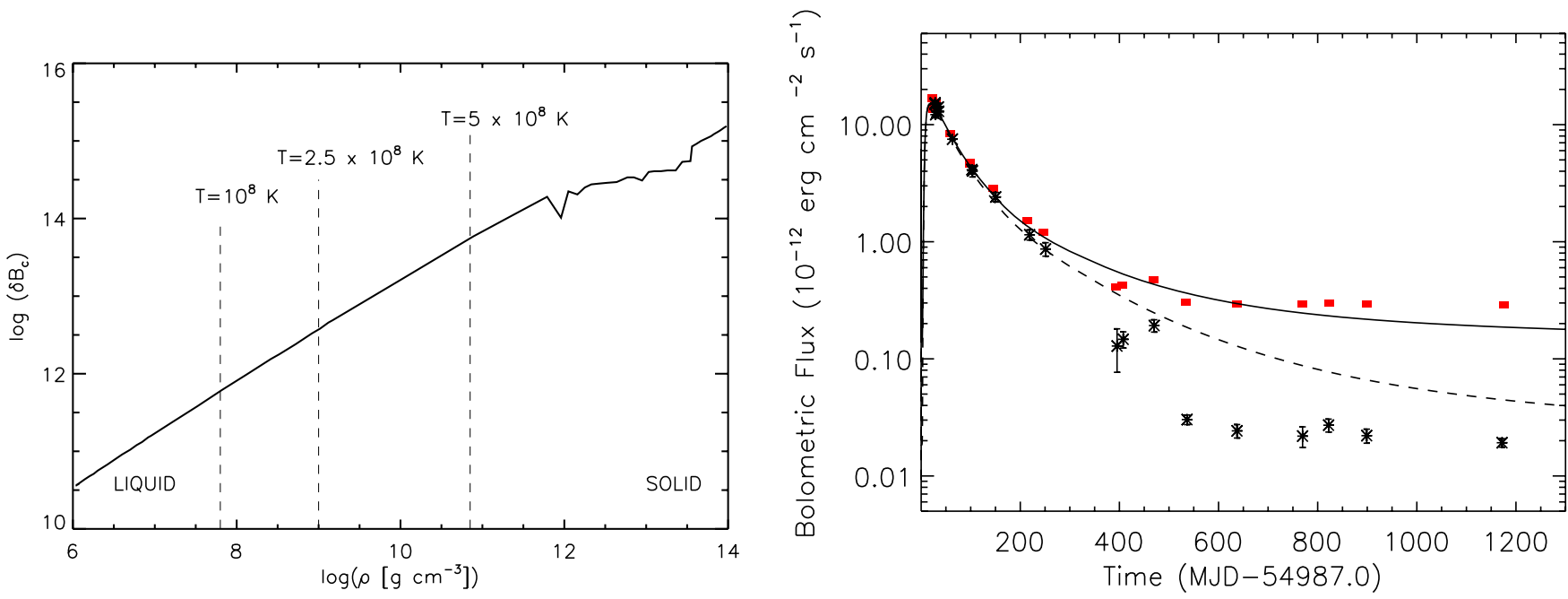

Figure 10. Left panel: minimum variation of the magnetic field required to break the crust by magnetic stresses as a function of density. The vertical dashed lines delimit the transition from solid to liquid for three different temperatures. Right panel: outburst modeling following Pons \& Rea (2012). Black data are the 0.5-10 keV unabsorbed flux, while red squares are the bolometric unabsorbed flux with the addition of the flux of a thermal component at $k T=0.05 \mathrm{keV}$ from the entire neutron star. Solid and dashed lines refer to the outburst model for the bolometric and $0.5-10 \mathrm{keV}$ thermal flux, respectively (see text for details).

(A color version of this figure is available in the online journal.) 
field variations $\delta B_{c} \gtrsim 10^{14} \mathrm{G}$ are required to fracture the crust. However, for old, cold neutron stars (crustal temperatures of $10^{8} \mathrm{~K}$ ), the solid crust extends down to $10^{8} \mathrm{~g} \mathrm{~cm}^{-3}$, and is much easier to break, even with variations of the magnetic field of the order of $\delta B_{c} \gtrsim 10^{12} \mathrm{G}$. Note also that fractures close to the inner crust are much more energetic (because of both the higher available elastic energy and larger volume involved) than fractures in the low density region. In the first case, one can reach up to $10^{44} \mathrm{erg}$, while in the second case, events of $\approx 10^{41} \mathrm{erg}$ are expected. A rough prediction of the expected outburst rate (Perna \& Pons 2011; Pons \& Perna 2011) for the solution model mentioned above gives $\lesssim 10^{-3}$ star-quakes $\mathrm{yr}^{-1}$ for an object as SGR $0418+5729$. Assuming that there are about $10^{4}$ neutron stars in the Galaxy with similar age, and that (approximatively) $10 \%$ of them are born as magnetars, a naive extrapolation of this event rate to the whole neutron star population leads to an expected low- $B$ magnetar outburst rate of $\lesssim 1$ per year. Therefore, we expect that more and more objects of this class will be discovered in the upcoming years (as, e.g., Swift 1822.3-1606; Rea et al. 2012; Scholz et al. 2012).

\subsection{SGR 0418+5729 Outburst Decay}

By modeling the flux evolution in time we tested if the crustal cooling model presented in Pons \& Rea (2012) can fit the flux decay of SGR $0418+5729$, on the wave of what done for Swift J1822.3-1606 (Rea et al. 2012). We assume a dipolar field of $6 \times 10^{12} \mathrm{G}$ (equatorial), an internal toroidal field of $10^{14} \mathrm{G}$ (at maximum) as inferred in Section 8.1, and an average surface temperature of $0.05 \mathrm{keV}$, which is the temperature we expect for the surface of such an old magnetar (note that in the $0.5-10 \mathrm{keV}$ band we are only seeing a tiny hot spot). The best modeling was found by injecting $2.5 \times 10^{26} \mathrm{erg} \mathrm{cm}^{-3}$ in a thin layer in the outer crust between $4.5 \times 10^{9}$ and $10^{10} \mathrm{~g} \mathrm{~cm}^{-3}$, and in the region contained within a cone with axis in the direction of the magnetic pole, and aperture $a \approx 0.4 \mathrm{rad}$, for a total energy deposition of $2.5 \times 10^{41}$ erg (compatible with typical magnetar outbursts; Pons \& Rea 2012). The evolution of the bolometric, and of the 0.5-10 keV flux is shown in Figure 10 (solid and dashed lines, respectively). We have shown with red squares how the observed flux decay would appear when adding the contribution of a blackbody component at $0.05 \mathrm{keV}$, mimicking the entire neutron star surface. It is clear that crustal cooling can easily explain the decay only if this further component is taken into account. In particular, the solid line is fitting the red points because the entire neutron star surface is taken into account, while it is not in the observed black data, which in fact cannot be reproduced by the dashed line. This is indicative of the difficulty of comparing theoretical cooling curves with data obtained in a certain energy band. We also note that no theoretical model predicts a surface temperature as high as $0.3 \mathrm{keV}$ on a timescale of years, unless a continuous energy release is assumed (e.g., by long-lived internal currents). We finally mention that all the previous considerations are based on the (implicit) assumption that the blackbody temperature is a measure of the physical temperature of the emitting region. If this turned out not to be the case, e.g., because the spectrum is thermal but not Planckian so that a color correction is required, the physical surface temperature may be smaller than the measured blackbody temperature.

An alternative model to the crustal cooling scenario consists in the presence of currents flowing into the magnetosphere through a gradually shrinking magnetic bundle heating the neutron star surface from the top. In particular, the deepest available XMM-Newton observation of SGR $0418+5729$, performed two
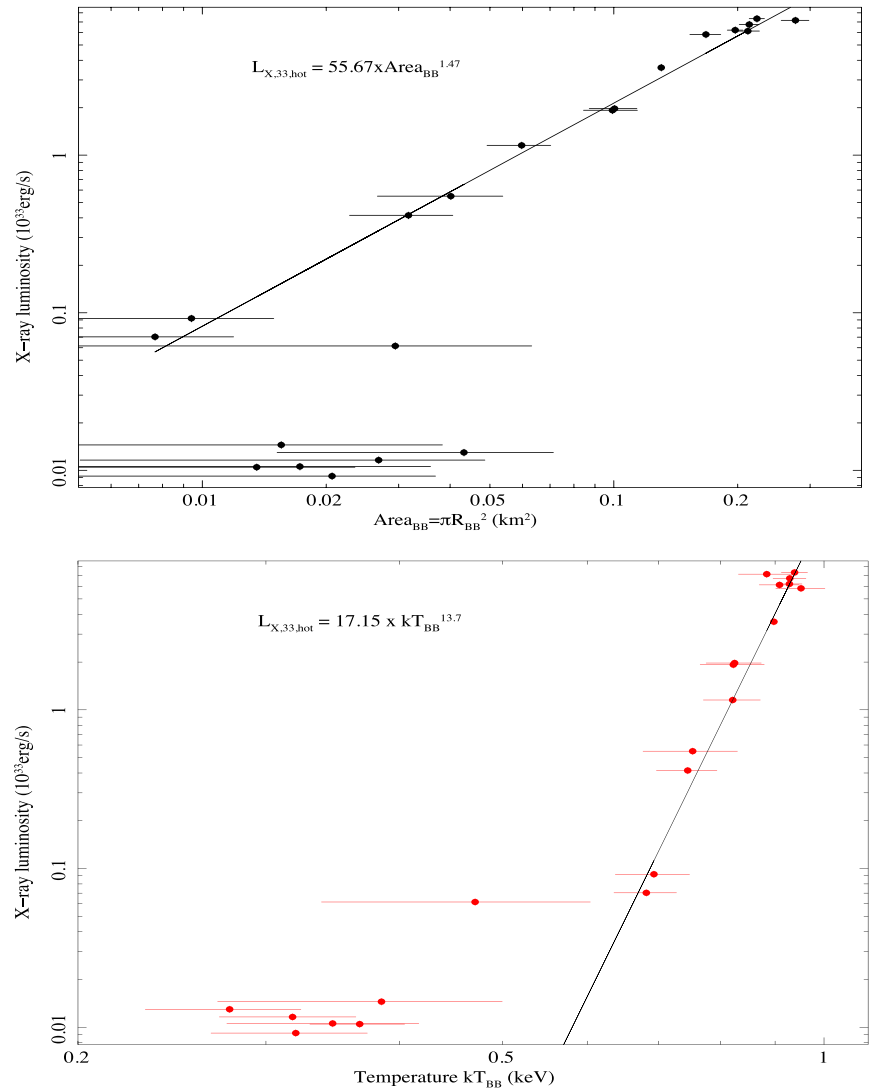

Figure 11. X-ray luminosity evolution as a function of the blackbody emitting area and temperature (see also Table 1; we assume a $2 \mathrm{kpc}$ distance).

(A color version of this figure is available in the online journal.)

months after the outburst onset, revealed that the $0.5-10 \mathrm{keV}$ spectrum of SGR $0418+5729$ is best reproduced by a blackbody component plus an additional non-thermal component, or by a resonant cyclotron scattering model. This suggests the presence of twisted magnetic field lines, at least in the first outburst stages. Furthermore, the limited spatial extent of the heated region $(<1 \mathrm{~km})$ is suggestive of a scenario in which the twist is confined within a small part of the magnetosphere, a thin current-carrying bundle, or $j$-bundle (Beloborodov 2009). As the $j$-bundle untwists during the outburst decay, the spectrum becomes more and more blackbody-like, as indeed observed.

Resonant cyclotron scattering from a thin, decaying $j$-bundle appears also capable of explaining (qualitatively) the spectrum and its evolution during the first outburst stages, but whether it can explain the double-peaked pulse profiles is unclear. However, this scenario has some further difficulties: (1) the total luminosity produced by currents in the bundle is, for such a low- $B$ and a small thermal spot, well below the one observed at early times, at least if the spot is at the polar cap (see again Beloborodov 2009 and also Turolla et al. 2011), (2) the timescale for the twist decay is much shorter $(<1 \mathrm{yr})$ than that implied by the long outburst of SGR $0418+5729$, and (3) an approximate relation between the emitting area and the luminosity exists ( $A \sim L^{2}$; Beloborodov 2009) if most of the luminosity is produced by current dissipation, but SGR $0418+5729$ data show a somewhat flatter dependence when the first stages of the outburst are fitted (see Figure 11).

In summary, the crustal cooling model, when including also a possible hidden contribution from the entire neutron star surface, appears favorable in explaining the outburst decay of 
SGR $0418+5729$. However, it is likely that a combination of crustal cooling and magnetospheric untwisting bundle can be operating at the first stages of the outburst.

\subsection{Constraints on the Presence of a Fossil Disk Surrounding SGR 0418+5729}

A fallback disk around SGR 0418+5729 was suggested by Alpar et al. (2011) as a way to aid the spin-down of the pulsar and explain the $9 \mathrm{~s}$ periodicity of this source. As an alternative, our results show (see Section 8.1) that both the thermal and the timing properties of this source can be reproduced for a pulsar age of $\sim 550 \mathrm{kyr}$ by properly accounting for magnetic field evolution and dissipation, which also imply that the neutron star was born with a much higher dipolar field than the one measured today (see Section 8.1). Hence, in principle, the timing properties of this source would not necessarily require an additional spin down torque by a disk. However, given the suggestion that fallback disks around isolated neutron stars might be common (Michel 1988; Chevalier 1989; Lin et al. 1991), it is worthwhile to use the current multi-band upper limits to set constraints on the presence of a fallback disk around SGR 0418+5729.

Since the pulsar is currently spinning down, any diskmagnetosphere interaction must probably occur in the propeller regime, with the pulsar transferring angular momentum to the disk. For this condition to be satisfied, the inner boundary of the disk, located at about the magnetospheric radius $R_{m}=$ $2.5 \times 10^{8}\left[\dot{M} /\left(10^{16} \mathrm{~g} \mathrm{~s}^{-1}\right)\right]^{-2 / 7}\left(M_{\mathrm{NS}} / M_{\odot}\right)^{-1 / 7}\left[B /\left(10^{12} \mathrm{G}\right)\right]^{4 / 7}$, must be equal to or larger than the corotation radius $R_{\mathrm{co}}=$ $\left(G M_{\mathrm{NS}}\right)^{1 / 3} \Omega^{2 / 3}$. The strongest constraint on the disk emission is obtained when the inner radius of the disk obtains its minimum value, i.e., $R_{\text {in }}=R_{m}=R_{\text {co }}$. For the outer radius, we assume $R_{\text {out }}=10^{14} \mathrm{~cm}$. We found that larger values do not result in appreciably larger emission at the frequency of interest, and hence this value allows to set the tightest constraint on the disk emission.

With the inner and outer disk radii fixed as discussed above, the emission spectra from the fallback disk is computed using the model of Perna et al. (2000). The disk is assumed to be optically thick and geometrically thin, and the anisotropy in the $\mathrm{X}$-ray luminosity from the source (which irradiates the disk) is neglected, since it is found to be of second order (Perna \& Hernquist 2000). The disk is assumed to be still "active," i.e., viscously accreting (see Menou et al. 2001). The disk emission is the result of both viscous dissipation and re-radiation of the pulsar X-ray luminosity. In order for the magnetospheric radius not to exceed the corotation radius, the accretion rate must be limited to $\dot{M} \lesssim 10^{15} \mathrm{~g} \mathrm{~s}^{-1}$. With this value, the disk luminosity in the millimeter band is dominated by reprocessing of the pulsar X-ray luminosity. At $166 \mathrm{GHz}$, the predicted flux is about $0.01 \mathrm{mJy}$ for a face-on disk, below the measured limit of $0.24 \mathrm{mJy}$. Hence the presence of a fossil disk cannot be ruled out by the current millimeter measurements. Even adding the contribution from the whole surface of the star by a putative thermal component at $0.05 \mathrm{keV}$, would bring the predicted millimeter flux just around the measured flux limit for a face-on disk.

The field around SGR 0418+5729 was also observed with the Grantecan and Hubble telescopes (Esposito et al. 2010; Durant et al. 2011). In particular, the latter observations were performed in two wide filters, the optical, with a pivot wavelength of $5921 \AA$, and in the NIR, with pivot wavelength of $11534 \AA$. The source was not detected down to the flux of
$f_{O}<2.3 \times 10^{-31} \mathrm{erg} \mathrm{s}^{-1} \mathrm{~cm}^{-2} \mathrm{~Hz}^{-1}$ and $f_{\mathrm{NIR}}<4.4 \times$ $10^{-31} \mathrm{erg} \mathrm{s}^{-1} \mathrm{~cm}^{-2} \mathrm{~Hz}^{-1}$, respectively. We found that this optical limit (nor the Grantecan or WHT limit) is not sufficiently constraining for a disk with the properties described above (the predicted emission for a face-on disk is about a factor of four below the limit). On the other hand, in the NIR, the observational limit is already able to rule out a face-on disk, which would yield an emission about twice larger than the measured flux limit. However, a disk inclined with respect to the observer by an angle $\cos \theta \lesssim 0.5$ would still be allowed by the observations (although falling short in explaining the X-ray bursts of this object).

\subsection{Conclusions}

At the time of this writing, in the ATNF pulsar catalogue (Manchester et al. 2005) 138 isolated radio pulsars have a dipolar magnetic field larger than that inferred for SGR 0418+5729. Our results imply that some of these objects might hide a strong toroidal component of the internal field, not measurable via the pulsar timing properties. A hint for such strong fields might be a high surface temperature, hotter than what would be predicted by standard cooling models at the pulsar age. However, only a few of those pulsars have had dedicated X-ray observations, and the shallow surveys do not suffice to detect such emission (expected to be as luminous as $L_{X} \sim 10^{31} \mathrm{erg} \mathrm{s}^{-1}$ ). Furthermore, our calculation of the outburst rate of a low magnetic field magnetar also suggests that roughly once a year a quiet neutron star might turn on with magnetar-like activity.

On the other hand, if indeed a large number of neutron stars is hiding a strong magnetic field component, there would be important consequences also for other branches of astrophysics. In particular, it would imply that supernova explosions should generally produce strong magnetic fields, and that most massive stars are either producing fast rotating cores during the explosion to activate a dynamo, or are strongly magnetized themselves. Furthermore, in this scenario a non-negligible fraction of gamma-ray bursts might be due to the formation of magnetars, and the gravitational wave background produced by magnetar births should then be larger than predicted so far (important for future instruments as the Advanced-LIGO).

We are indebted to the Chandra, Swift, XMM-Newton, GBT, WHT, and PdBI support teams, with a special thank to Michael Bremer, for the extraordinary job in planning the PdB observations presented in this paper. We thank S. Mereghetti and H. Tong for their valuable comments on the manuscript. N.R. is supported by a Ramon y Cajal Research Fellowship, and by grants AYA2009-07391, AYA2012-39303, SGR2009-811, TW2010005, and iLINK 2011-0303. J.A.P. and D.V. acknowledge support from the grants AYA 2010-21097-C03-02 and Prometeo/2009/103. R.T. and S.M. are partially funded through an INAF 2011 PRIN grant. A.P. is supported by a JAE-Doc CSIC fellowship co-funded with the European Social Fund under the program "Junta para la Ampliación de Estudios," by the Spanish MICINN grant AYA2011-30228-C03-02 (co-funded with FEDER funds), and by the AGAUR grant 2009SGR1172 (Catalonia).

\section{REFERENCES}

Abdo, A. A., Ackermann, M., Ajello, M., et al. 2010, ApJL, 725, L73 Aguilera, D. N., Pons, J. A., \& Miralles, J. A. 2008, A\&A, 486, 255 Alpar, M. A., Ertan, Ü., \& Çalışkan, Ş. 2011, ApJL, 732, L4 
Anders, E., \& Grevesse, N. 1989, GeCoA, 53, 197

Atwood, W. B., Abdo, A. A., Ackermann, M., et al. 2009, ApJ, 697, 1071

Balucinska-Church, M., \& McCammon, D. 1992, ApJ, 400, 699

Beloborodov, A. M. 2009, ApJ, 703, 1044

Burrows, D. N., Hill, J. E., Nousek, J. A., et al. 2005, SSRv, 120, 165

Chevalier, R. A. 1989, ApJ, 346, 847

Condon, J. J., Cotton, W. D., Greisen, E. W., et al. 1998, AJ, 115, 1693

Dall'Osso, S., Israel, G. L., Stella, L., Possenti, A., \& Perozzi, E. 2003, ApJ, 599,485

Duncan, R. C., \& Thompson, C. 1992, ApJL, 392, L9

DuPlain, R., Ransom, S., Demorest, P., et al. 2008, Proc. SPIE, 7019, 45

Durant, M., Kargaltsev, O., \& Pavlov, G. G. 2011, ApJ, 742, 77

Esposito, P., Israel, G. L., Turolla, R., et al. 2010, MNRAS, 405, 1787

Esposito, P., Israel, G. L., Turolla, R., et al. 2011, MNRAS, 416, 205

Ferrario, L., \& Wickramasinghe, D. 2006, MNRAS, 367, 1323

Garmire, G. P., Bautz, M. W., Ford, P. G., et al. 2003, Proc. SPIE, 4851, 28

Glampedakis, K., Jones, D. I., \& Samuelsson, L. 2011, MNRAS, 413, 2021

Jahoda, K., Swank, J. H., Giles, A. B., et al. 1996, Proc. SPIE, 2808, 59

Jansen, F., Lumb, D., Altieri, B., et al. 2001, A\&A, 365, L1

Lin, D. N. C., Woosley, S. E., \& Bodenheimer, P. H. 1991, Natur, 353, 827

Lorimer, D. R., Edel, S., Kondratiev, V. I., et al. 2009, ATel, 2096, 1

Malheiro, M., Rueda, J. A., \& Ruffini, R. 2012, PASJ, 64, 56

Manchester, R. N., Hobbs, G. B., Teoh, A., \& Hobbs, M. 2005, AJ, 129,1993

Mattox, J. R., Bertsch, D. L., Chiang, J., et al. 1996, ApJ, 461, 396

Menou, K., Perna, R., \& Hernquist, L. 2001, ApJ, 559, 1032

Mereghetti, S. 2008, A\&ARv, 15, 225

Michel, F. C. 1988, Natur, 333, 644

Nobili, L., Turolla, R., \& Zane, S. 2008, MNRAS, 386, 1527

Ouyed, R., Leahy, D., \& Niebergal, B. 2011, MNRAS, 415, 1590

Perna, R., \& Hernquist, L. 2000, ApJL, 544, L57

Perna, R., Hernquist, L., \& Narayan, R. 2000, ApJ, 541, 344

Perna, R., \& Pons, J. A. 2011, ApJL, 727, L51

Pons, J. A., Miralles, J. A., \& Geppert, U. 2009, A\&A, 496, 207
Pons, J. A., \& Perna, R. 2011, ApJ, 741, 123

Pons, J. A., \& Rea, N. 2012, ApJL, 750, L6

Protassov, R., van Dyk, D. A., Connors, A., Kashyap, V. L., \& Siemiginowska, A. 2002, ApJ, 571, 545

Rea, N. 2013, in IAU Symp. 291, Neutron Stars and Pulsars: Challenges and Opportunities after 80 Years, ed. J. van Leeuwen (Cambridge: Cambridge Univ. Press), 11

Rea, N., \& Esposito, P. 2011, in Astrophysics and Space Science Proceedings, High-energy Emission from Pulsars and Their Systems, ed. D. F. Torres \& N. Rea (Berlin: Springer), 247

Rea, N., Esposito, P., Turolla, R., et al. 2010, Sci, 330, 944

Rea, N., Israel, G. L., Esposito, P., et al. 2012, ApJ, 754, 27

Rea, N., Israel, G. L., Turolla, R., et al. 2009, MNRAS, 396, 2419

Rea, N., Zane, S., Lyutikov, M., \& Turolla, R. 2007, Ap\&SS, 308, 61

Rea, N., Zane, S., Turolla, R., Lyutikov, M., \& Götz, D. 2008, ApJ, 686, 1245

Scholz, P., Ng, C.-Y., Livingstone, M. A., et al. 2012, ApJ, 761, 66

Soni, V. 2012, MNRAS, 425, L15

Spitkovsky, A. 2006, ApJL, 648, L51

Strüder, L., Briel, U., Dennerl, K., et al. 2001, A\&A, 365, L18

Thompson, C., \& Duncan, R. C. 1993, ApJ, 408, 194

Thompson, C., \& Duncan, R. C. 1995, MNRAS, 275, 255

Thompson, C., Lyutikov, M., \& Kulkarni, S. R. 2002, ApJ, 574, 332

Tong, H., \& Xu, R. X. 2012, ApJL, 757, L10

Turner, M. J. L., Abbey, A., Arnaud, M., et al. 2001, A\&A, 365, L27

Turolla, R., Zane, S., Pons, J. A., Esposito, P., \& Rea, N. 2011, ApJ, 740, 105

van der Horst, A. J., Connaughton, V., Kouveliotou, C., et al. 2010, ApJL, 711, L1

van Straten, W., \& Bailes, M. 2010, in Astrophysics Source Code Library, record: ascl1010.006

Viganò, D., Rea, N., Pons, J. A., et al. 2013, MNRAS, submitted

Wachter, S., Gelino, C. R., Gelino, D. M., et al. 2009, ATel, 2164

Zane, S., Rea, N., Turolla, R., \& Nobili, L. 2009, MNRAS, 398, 1403

Zombeck, M. V., Chappell, J. H., Kenter, A. T., et al. 1995, Proc. SPIE, 2518, 96 